\title{
Tree expansion in time-dependent perturbation theory
}

\author{
Christian Brouder and Ângela Mestre \\ Institut de Minéralogie et de Physique des Milieux Condensés, CNRS UMR 7590, \\ Universités Paris 6 et 7, IPGP, 140 rue de Lourmel, 75015 Paris, France. \\ Frédéric Patras \\ Laboratoire J.-A. Dieudonné, CNRS UMR 6621, \\ Université de Nice, Parc Valrose, 06108 Nice Cedex 02, France.
}

(Dated: October 16, 2018)

\begin{abstract}
The computational complexity of time-dependent perturbation theory is well-known to be largely combinatorial whatever the chosen expansion method and family of parameters (combinatorial sequences, Goldstone and other Feynman-type diagrams...). We show that a very efficient perturbative expansion, both for theoretical and numerical purposes, can be obtained through an original parametrization by trees and generalized iterated integrals. We emphasize above all the simplicity and naturality of the new approach that links perturbation theory with classical and recent results in enumerative and algebraic combinatorics. These tools are applied to the adiabatic approximation and the effective Hamiltonian. We prove perturbatively and non-perturbatively the convergence of Morita's generalization of the Gell-Mann and Low wavefunction. We show that summing all the terms associated to the same tree leads to an utter simplification where the sum is simpler than any of its terms. Finally, we recover the time-independent equation for the wave operator and we give an explicit non-recursive expression for the term corresponding to an arbitrary tree.
\end{abstract}

\section{INTRODUCTION}

Effective Hamiltonians provide a way to determine the low-energy eigenvalues of a (possibly infinite dimensional) Hamiltonian by diagonalizing a matrix defined in a subspace of small dimension, called the model space and hereafter denoted by $M$. Because of this appealing feature, effective Hamiltonians are used in nuclear, atomic, molecular, chemical and solid-state physics 1 .

These theories are plagued with a tremendous combinatorial complexity because of the presence of folded diagrams (to avoid singularities of the adiabatic limit), partial resummations, subtle "linkedness" properties and the exponential growth of the number of graphs with the order of perturbation. This complexity has two consequences: on the one hand, few results are proved in the mathematical sense of the word, on the other hand, it is difficult to see what is the underlying structure of the perturbative expansion that could lead to useful resummations and non-perturbative approximations.

To avoid these pitfalls, we take a bird's-eye view of the problem and consider a general time-dependent Hamiltonian $H(t)$. This way, we disentangle the problem from the various particular forms that can be given to the Hamiltonian and which have lead in the past to various perturbative expansions. To be precise, take the example of fermions in molecular systems. The Coulomb interaction between the electrons (say $V$ ) can be viewed as a perturbation of a "free Hamiltonian" modeling the interaction with the nuclei (in the Born-Oppenheimer approximation). One can take advantage of the particular form of $V$ (which is a linear combination of products of two creation and two annihilation operators in the second quantization picture) to represent the perturbative expansions using a given family of Goldstone diagrams (see e.g. ref. 2 for such a family). However, the general results on perturbative expansions (such as the convergence of the time-dependent wave operator) do not depend on such a particular choice.

Thus, we consider an Hamiltonian $H(t)$ and we build its evolution operator $U\left(t, t_{0}\right)$, which is the solution of the Schrödinger equation (in units $\hbar=1$ )

$$
\imath \frac{\partial U\left(t, t_{0}\right)}{\partial t}=H(t) U\left(t, t_{0}\right),
$$

with the boundary condition $U\left(t_{0}, t_{0}\right)=1$. In perturbation theory, $H(t):=\mathrm{e}^{-\epsilon|t|} \mathrm{e}^{\imath H_{0} t} V \mathrm{e}^{-\imath H_{0} t}$ is the adiabatically switched interaction Hamiltonian in the interaction picture (here $H_{0}$ and $V$ stand respectively for the "free" and interaction terms of the initial Hamiltonian) and singularities show up in the adiabatic limit $\left(t_{0} \rightarrow-\infty\right.$ and $\left.\epsilon \rightarrow 0\right)$. Morita discovered ${ }^{2}$ that, in this setting, the time-dependent wave operator

$$
\Omega\left(t, t_{0}\right):=U\left(t, t_{0}\right) P\left(P U\left(t, t_{0}\right) P\right)^{-1},
$$

where $P$ is the projection onto the model space $M$, has no singularity in the adiabatic limit. Moreover, the wave 
operator determines the effective Hamiltonian because

$$
H_{\mathrm{eff}}:=\lim _{\epsilon \rightarrow 0} P H \Omega(0,-\infty) .
$$

However, as we have already alluded to, the effective computation of these operators raises several combinatorial and analytical problems that have been addressed in a long series of articles (several of which will be referred to in the present article).

In the first sections of the paper, we consider a general time-dependent Hamiltonian $H(t)$ (not necessarily in the interaction picture). In this broader setting, Jolicard ${ }^{3}$ found that the time-dependent wave operator provides also a powerful description of the evolution of quantum systems (see ref. 4 for applications). Then, we derive three (rigorously proven) series expansions of the wave operator. The first one is classical and can be physically interpreted as the replacement of causality (i.e. the Heaviside step function $\left.\theta\left(t-t^{\prime}\right)\right)$ by a "propagator" $\theta_{P}\left(t-t^{\prime}\right):=\theta\left(t-t^{\prime}\right) Q-\theta\left(t^{\prime}-t\right) P$, where $Q=1-P$. This "propagator" is causal out of the model space $\left(\theta_{P}\left(t-t^{\prime}\right)=0\right.$ for $t<t^{\prime}$ on the image of $\left.Q\right)$ and anticausal on it, like the Feynman propagator of quantum field theory $\underline{\underline{\underline{5}}}$. However, this sum of causal and anticausal orderings is cumbersome to use in practice. A second series expansion is obtained by writing the wave operator as a sum of integrals over all possible time orderings of the Hamiltonians $H\left(t_{i}\right)$ (see Sect. IIB). This expansion, parametrized by all the permutations (or equivalent families), is used in many-body theory and gives rise to a large number of complicated terms. The third expansion is obtained by noticing that some time orderings can be added to give simpler expressions. This series is naturally indexed by trees and is the main new tool developed in the present paper. Among others, we derive a very simple recurrence relation for the terms of the series. We also show that the very structure of the corresponding generalized iterated integrals showing up in the expansion is interesting on its own. These integrals carry naturally a rich algebraic structure that is connected to several recent results in the field of combinatorial Hopf algebras and noncommutative symmetric functions. The corresponding algebraic results that point out in the direction of the existence of a specific Lie theory for effective Hamiltonians (generalizing the usual Lie theory) are gathered in an Appendix.

In the last sections of the paper, we restrict $H(t)$ to the interaction picture and we consider the adiabatic limit. We first prove that the adiabatic limit exists non perturbatively. We show that the effective Hamiltonian defined by eq. (2) has the expected properties. Then, we expand the series and we give a rigorous (but lengthy) proof that the term corresponding to each time ordering has an adiabatic limit. Then, we consider the series indexed by trees and we give a short and easy proof of the existence of that limit. Finally, we provide a direct rule to calculate the term corresponding to a given tree and establish the connection between the time-dependent approach and the time-independent equations discovered by Lindgren $\underline{6}$ and Kvasnička ${ }^{7}$.

The existence of this series indexed by trees can be useful in many ways: (i) It describes a sort of superstructure that is common to all many-body theories without knowing the exact form of the interaction Hamiltonian; (ii) It considerably simplifies the manipulation of the general term of the series by providing a powerful recurrence relation; (iii) It provides simple algorithms to calculate the terms of the series; (iv) The number of trees of order $n$, $\frac{1}{n+1}\left(\begin{array}{c}2 n \\ n\end{array}\right) \approx \frac{4^{n}}{n^{\frac{3}{2}} \sqrt{\pi}}$ being subexponential, it improves the convergence of the series $\stackrel{8,9}{\text {; }}$ (v) It can deal with problems where the Hamiltonian $H_{0}$ is not quadratic. Indeed, many-body theories most often require the Hamiltonian $H_{0}$ to be free, i.e. to be a quadratic function of the fields 10 . As noticed by Bulaevskii11, this is not the good point of view for some applications. For example, in the microscopic theory of the interaction of radiation with matter, it is natural to take for $H_{0}$ the Hamiltonian describing electrons and nuclei in Coulomb interaction ${ }^{12}$, the perturbation being the interaction with the transverse electric field. In that case, quadratic free Hamiltonians many-body theories break down whereas our approach is still valid. Actually, it is precisely for that reason that we originally developed the tree series approach; (vi) Last, but not least, the tree-theoretical approach connects many-body theories with a large field of knowledge that originates in the "birth of modern bijective combinatorics" in the seventies with in particular the seminal works of Foata, Schützenberger and Viennot 13 ,14 . See e.g. ref. 15 for a survey of the modern combinatorial theory of tree-like structures.

From the physical point of view, the tree expansion is particularly interesting in the adiabatic limit. Indeed, the denominator of each of its terms is a product of $E_{i}^{Q}-E_{j}^{P}$ factors, where $E_{j}^{P}$ is the energy of a state in the model space $M$ and $E_{j}^{Q}$ the energy of a state not belonging to $M$. In the usual many-body expansions, the denominators are products of $\sum_{i} E_{i}^{Q}-\sum_{j} E_{j}^{P}$ factors, where the sums contain various numbers of elements (corresponding therefore to multiple transitions between low-energy and excitated levels). In that sense, the tree expansion is the simplest possible because each term is a product of single transitions between two states.

We now list the main new results of this paper: (i) A recursion formula that generates the simplified terms of the time-dependent perturbation series (theorem 5); (ii) when the interaction is adiabatically switched on, a nonperturbative proof of the convergence of the wave operator and a characterization of the states of the model space that are transformed into eigenstates of $H$ by the wave operator (theorem [6); (iii) a proof of the existence of the 
adiabatic limit for the terms of the series expansion of the wave operator (theorem 91); (iv) a recursive formula (lemma 13) and an explicit form (theorem 14) for the general term of the time-independent perturbation series.

\section{TIME-DEPENDENT HAMILTONIAN AND COMBINATORICS}

We consider a time-dependent Hamiltonian $H(t)$, which is a self-adjoint operator on a Hilbert space $\mathcal{H}$, and its evolution operator $U\left(t, t_{0}\right)$ defined in eq. (1). Since we are interested in the combinatorial aspects of the problem, we consider the simple case where $H(t)$ is a strongly continuous map from $\mathbb{R}$ into the bounded self-adjoint operators on $\mathcal{H}^{16}$. In that case, the Picard-Dyson series

$$
1+\sum_{n=1}^{\infty}(-\imath)^{n} \int_{t_{0}}^{t} \mathrm{~d} t_{1} \int_{t_{0}}^{t_{1}} \mathrm{~d} t_{2} \ldots \int_{t_{0}}^{t_{n-1}} \mathrm{~d} t_{n} H\left(t_{1}\right) \ldots H\left(t_{n}\right)
$$

converges in the uniform operator topology to $U\left(t, t_{0}\right)$ and $U\left(t, t_{0}\right)$ is a jointly strongly continuous two-parameter family of unitaries on $\mathcal{H}$ (see section X.12 of ref. 17).

Following Morita ${ }^{2}$, Jolicard ${ }^{3}$ established a connection between the evolution operator and the effective Hamiltonian approach by defining

$$
\Omega\left(t, t_{0}\right):=U\left(t, t_{0}\right) P\left(P U\left(t, t_{0}\right) P\right)^{-1}
$$

where $P$ is a projection operator onto $M$ and $\left(P U\left(t, t_{0}\right) P\right)^{-1}$ is the inverse of $P U\left(t, t_{0}\right) P$ as a map from $M=P \mathcal{H}$ to itself. This map is invertible if and only if there is no state $|\phi\rangle$ in $M$ such that $\langle\phi| U\left(t, t_{0}\right) P=0$. This condition is similar to the one of time-independent perturbation theory ${ }^{18}$. We assume from now on that the condition is satisfied and we define three expansions for $\Omega\left(t, t_{0}\right)$.

\section{A. First expression for $\Omega$}

We start by proving an elegant expression for $\Omega$, that was stated by Michels and Suttorp 19 and Dmitriev and Solnyshkina 20 .

\section{Theorem 1}

$$
\Omega\left(t, t_{0}\right)=P+Q \sum_{n=1}^{\infty}(-\imath)^{n} \int_{t_{0}}^{t} \mathrm{~d} t_{1} \int_{t_{0}}^{t} \mathrm{~d} t_{2} \ldots \int_{t_{0}}^{t} \mathrm{~d} t_{n} H\left(t_{1}\right) \theta_{P}\left(t_{1}-t_{2}\right) H\left(t_{2}\right) \ldots \theta_{P}\left(t_{n-1}-t_{n}\right) H\left(t_{n}\right) P
$$

where $Q=1-P$ and $\theta_{P}(t)=\theta(t) Q-\theta(-t) P$, with $\theta$ the Heaviside step function.

Proof. We first rewrite the Picard-Dyson series as $U\left(t, t_{0}\right)=1+\sum_{n} U_{n}\left(t, t_{0}\right)$ with $U_{1}\left(t, t_{0}\right):=-\imath \int_{t_{0}}^{t} \mathrm{~d} t_{1} H\left(t_{1}\right)$ and, for $n>1$,

$$
U_{n}\left(t, t_{0}\right):=(-\imath)^{n} \int_{t_{0}}^{t} \mathrm{~d} t_{1} \ldots \int_{t_{0}}^{t} \mathrm{~d} t_{n} H\left(t_{1}\right) \theta\left(t_{1}-t_{2}\right) \ldots \theta\left(t_{n-1}-t_{n}\right) H\left(t_{n}\right) .
$$

Then, by using $\theta(t)+\theta(-t)=1$, we notice that

$$
\theta(t)=P+\theta(t)-\theta(t) P-\theta(-t) P=P+\theta_{P}(t) .
$$

Now, we replace $\theta(t)$ by the sum of operators $P+\theta_{P}(t)$ in the expression for $U_{n}\left(t, t_{0}\right)$. This gives us $2^{n-1}$ terms with various numbers of $P$ and $\theta_{P}$. Denote by $C_{n}\left(t, t_{0}\right)$ the term with no $P$ (with the particular case $C_{1}\left(t, t_{0}\right)=U_{1}\left(t, t_{0}\right)$ ). Take then any other term. There is an index $i$ such that the first $P$ from the left occurs after $H\left(t_{i}\right)$. Therefore, the integrand of this term is

$$
H\left(t_{1}\right) \theta_{P}\left(t_{1}-t_{2}\right) \ldots \theta_{P}\left(t_{i-1}-t_{i}\right) H\left(t_{i}\right) P H\left(t_{i+1}\right) \ldots
$$

Observe that the integral over $t_{1}, \ldots, t_{i}$ is independent from the integral over $t_{i+1}, \ldots, t_{n}$. The first integral gives $C_{i}\left(t, t_{0}\right)$, the second integral is a term of the Picard-Dyson series for $U_{n-i}\left(t, t_{0}\right)$. Thus, the sum of the $2^{n-1}$ terms yields

$$
U_{n}\left(t, t_{0}\right)=C_{n}\left(t, t_{0}\right)+\sum_{i=1}^{n-1} C_{i}\left(t, t_{0}\right) P U_{n-i}\left(t, t_{0}\right)
$$


If we denote by $K\left(t, t_{0}\right)$ the sum of all the $C_{n}\left(t, t_{0}\right)$ with $n>0$, we obtain $U=1+K+K P(U-1)$, so that

$$
U P=P+K P U P \text {. }
$$

The operator $K$ is called the reduced evolution operator by Lindgren and collaborators 21 . If we define $\omega:=P+Q K P$, eq. (4) becomes

$$
U P=P+(\omega-P+P K P) P U P=P+\omega P U P-P U P+P K P U P .
$$

This equation can be simplified by using eq. (41) again

$$
U P=P+\omega P U P-P P=\omega P U P .
$$

Thus, $\omega=\Omega$ and eq. (3) is satisfied.

Despite its elegance, eq. (3) is not immediately usable. To illustrate this point, consider the third-order term

$$
\Omega_{3}=\imath Q \int_{t_{0}}^{t} \mathrm{~d} t_{1} \int_{t_{0}}^{t} \mathrm{~d} t_{2} \int_{t_{0}}^{t} \mathrm{~d} t_{3} H\left(t_{1}\right) \theta_{P}\left(t_{1}-t_{2}\right) H\left(t_{2}\right) \theta_{P}\left(t_{2}-t_{3}\right) H\left(t_{3}\right) P .
$$

If we expand $\theta_{P}(t)=\theta(t) Q-\theta(-t) P$, we obtain four terms

$$
\begin{aligned}
\imath Q H\left(t_{1}\right) Q H\left(t_{2}\right) Q H\left(t_{3}\right) P & \text { for } t_{1} \geq t_{2} \text { and } t_{2} \geq t_{3}, \\
-\imath Q H\left(t_{1}\right) Q H\left(t_{2}\right) P H\left(t_{3}\right) P & \text { for } t_{1} \geq t_{2} \text { and } t_{2} \leq t_{3}, \\
-\imath Q H\left(t_{1}\right) P H\left(t_{2}\right) Q H\left(t_{3}\right) P & \text { for } t_{1} \leq t_{2} \text { and } t_{2} \geq t_{3}, \\
\imath Q H\left(t_{1}\right) P H\left(t_{2}\right) P H\left(t_{3}\right) P & \text { for } t_{1} \leq t_{2} \text { and } t_{2} \leq t_{3} .
\end{aligned}
$$

The first and last terms have integration range $t_{1} \geq t_{2} \geq t_{3}$ and $t_{3} \geq t_{2} \geq t_{1}$, respectively and give rise to iterated integrals. The integration range of the second term is $t_{1} \geq t_{2}$ and $t_{2} \leq t_{3}$. Such an integration range is not convenient because the relative position of $t_{1}$ and $t_{3}$ is not specified. The integration range has to be split into the two subranges $t_{1} \geq t_{3} \geq t_{2}$ and $t_{3} \geq t_{1} \geq t_{2}$. Each subrange defines now an iterated integral. For example $t_{1} \geq t_{3} \geq t_{2}$ gives

$$
-\imath \int_{t_{0}}^{t} \mathrm{~d} t_{1} \int_{t_{0}}^{t_{1}} \mathrm{~d} t_{3} \int_{t_{0}}^{t_{3}} \mathrm{~d} t_{2} Q H\left(t_{1}\right) Q H\left(t_{2}\right) P H\left(t_{3}\right) P .
$$

Similarly, the integration range of the third term $\left(t_{1} \leq t_{2}\right.$ and $\left.t_{2} \geq t_{3}\right)$ is the union of $t_{2} \geq t_{1} \geq t_{3}$ and $t_{2} \geq t_{3} \geq t_{1}$. We see that $\Omega_{3}$ is sum of six iterated integrals corresponding to the six possible orderings of $t_{1}, t_{2}$ and $t_{3}$.

\section{B. $\Omega$ in terms of permutations}

We consider again the previous example, and we change variables to have a fixed integration range $s_{1} \geq s_{2} \geq s_{3}$. If we sum over all time orderings, we obtain

$$
\begin{aligned}
\Omega_{3}= & \imath \int_{t_{0}}^{t} \mathrm{~d} s_{1} \int_{t_{0}}^{s_{1}} \mathrm{~d} s_{2} \int_{t_{0}}^{s_{2}} \mathrm{~d} s_{3}\left(Q H\left(s_{1}\right) Q H\left(s_{2}\right) Q H\left(s_{3}\right) P-Q H\left(s_{1}\right) Q H\left(s_{3}\right) P H\left(s_{2}\right) P-Q H\left(s_{2}\right) Q H\left(s_{3}\right) P H\left(s_{1}\right) P\right. \\
& \left.-Q H\left(s_{2}\right) P H\left(s_{1}\right) Q H\left(s_{3}\right) P-Q H\left(s_{3}\right) P H\left(s_{1}\right) Q H\left(s_{2}\right) P+Q H\left(s_{3}\right) P H\left(s_{2}\right) P H\left(s_{1}\right) P\right) .
\end{aligned}
$$

In ref. 22, we showed that this result can be generalized to all orders and that $\Omega_{n}$ is a sum of $n$ ! iterated integrals corresponding to all the orderings of $t_{1}, \ldots, t_{n}$. More precisely, we obtained the series expansion for $\Omega$

$$
\Omega\left(t, t_{0}\right)=P+\sum_{n=1}^{\infty} \sum_{\sigma \in \mathcal{S}_{n}} \int_{t_{0}}^{t} \mathrm{~d} t_{1} \int_{t_{0}}^{t_{1}} \mathrm{~d} t_{2} \ldots \int_{t_{0}}^{t_{n-1}} \mathrm{~d} t_{n} X\left(t_{\sigma(1)}\right) \ldots X\left(t_{\sigma(n)}\right)
$$

where $\mathcal{S}_{n}$ is the group of permutations of $n$ elements. The operators $X$ are defined, for $n=1$, by $X(t):=-\imath Q H(t) P$ and, for $n>1$ and any $\sigma \in \mathcal{S}_{n}$, by $X\left(t_{\sigma(1)}\right):=-\imath Q H\left(t_{\sigma(1)}\right)$ and

$$
\begin{aligned}
& X\left(t_{\sigma(p)}\right):=-\imath Q H\left(t_{\sigma(p)}\right) \text { if } 1<p<n \text { and } \sigma(p)>\sigma(p-1), \\
& X\left(t_{\sigma(p)}\right):=\imath P H\left(t_{\sigma(p)}\right) \text { if } 1<p<n \text { and } \sigma(p)<\sigma(p-1), \\
& X\left(t_{\sigma(n)}\right):=-\imath Q H\left(t_{\sigma(n)}\right) P \text { if } \sigma(n)>\sigma(n-1), \\
& X\left(t_{\sigma(n)}\right):=\imath P H\left(t_{\sigma(n)}\right) P \text { if } \sigma(n)<\sigma(n-1) .
\end{aligned}
$$


Each term of eq. (6) is now written as an iterated integral. However, the expansion (6) is still not optimal because some of its terms can be summed to get a simpler expression. As an example, consider the fourth and fifth terms of eq. (5), where we replace $s_{i}$ by $t_{i}$.

$$
Z:=-\imath \int_{t_{0}}^{t} \mathrm{~d} t_{1} \int_{t_{0}}^{t_{1}} \mathrm{~d} t_{2} \int_{t_{0}}^{t_{2}} \mathrm{~d} t_{3}\left(Q H\left(t_{2}\right) P H\left(t_{1}\right) Q H\left(t_{3}\right) P+Q H\left(t_{3}\right) P H\left(t_{1}\right) Q H\left(t_{2}\right) P\right) .
$$

The first and second terms of the right hand side of this equation are denoted by $Z_{1}$ and $Z_{2}$, respectively. We transform $Z_{2}$ by exchanging variables $t_{2}$ and $t_{3}$.

$$
Z_{2}=-\imath \int_{t_{0}}^{t} \mathrm{~d} t_{1} \int_{t_{0}}^{t_{1}} \mathrm{~d} t_{3} \int_{t_{0}}^{t_{3}} \mathrm{~d} t_{2} Q H\left(t_{2}\right) P H\left(t_{1}\right) Q H\left(t_{3}\right) P=-\imath \int_{t_{0}}^{t} \mathrm{~d} t_{1} \int_{t_{0}}^{t_{1}} \mathrm{~d} t_{2} \int_{t_{2}}^{t_{1}} \mathrm{~d} t_{3} Q H\left(t_{2}\right) P H\left(t_{1}\right) Q H\left(t_{3}\right) P
$$

where we also exchanged the order of the integrations over $t_{3}$ and $t_{2}$. This can be added to $Z_{1}$ and we obtain the simpler expression

$$
Z=-\imath \int_{t_{0}}^{t} \mathrm{~d} t_{1} \int_{t_{0}}^{t_{1}} \mathrm{~d} t_{2} \int_{t_{0}}^{t_{1}} \mathrm{~d} t_{3} Q H\left(t_{2}\right) P H\left(t_{1}\right) Q H\left(t_{3}\right) P
$$

Such a simplification is not possible for the other terms of $\Omega_{3}$. In the next section, we determine how this simplification can be extended to the general term of $\Omega$.

Before closing this section, we need to specify more precisely the relation between the permutations $\sigma$ and the sequence of $P$ and $Q$ in the expansion of eq. (3). When we expand all the $\theta_{P}\left(t_{i}-t_{i+1}\right)$ in eq. (3), we obtain an integrand of the form $(-\imath)^{n} Q H\left(t_{1}\right) R_{1} H\left(t_{2}\right) \ldots R_{n-1} H\left(t_{n}\right) P$, multiplied by a product of Heaviside functions, where $R_{i}$ takes the value $-P$ or $Q$. We aim to determine the relation between the sequence $R_{1} \ldots R_{n-1}$ and the permutations $\sigma$ in eq. (6). From the definition of $X\left(t_{\sigma(i)}\right)$, it appears that $R_{i}=-P$ if $\sigma(i)>\sigma(i+1)$ and $R_{i}=Q$ if $\sigma(i)<\sigma(i+1)$. The set of indices $i$ such that $\sigma(i)>\sigma(i+1)$ is called the descent set of $\sigma$, denoted by $D_{\sigma}$. It is also called the shape of the permutation ${ }^{23}$. For instance, the descent set of the permutations (213) and (312) is $\{1\}$, corresponding to $\left(R_{1}, R_{2}\right)=(-P, Q)$.

\section{Permutations and trees}

In many-body physics, the expansion in Goldstone diagrams corresponds (among other things) to the expansion of $\Omega$ into all time orderings $\Omega_{\sigma}$. In that context, several authors noticed that some diagrams corresponding to different orderings can be added to give a simple sum ${ }^{1,19,24}-27$, as we saw in the previous section. These are special cases of the simplification that we shall present which, as far as we know, was never stated in full generality. The first difficulty is to find the proper combinatorial object to represent the sets of permutations that lead to simplified sums. We shall find it after a short algebraic detour meant to recall the notion of tree and its relation to permutations 13.23 . The trees we consider have received various names in the literature: binary trees in ref. 23, but also (to quote only a few occurrences) plane rooted complete binary tree ${ }^{28}$, extended binary trees ${ }^{29}$ or planar binary trees ${ }^{30}$. Since these objects are rarely used in physics, we first link them with the trees commonly found in graph theory. This lengthy definition will then be replaced by a much easier one.

A common tree is a connected graph without loops. In other words, a common tree is a set of vertices linked by edges such that there is a unique way to go from any vertex to any another one by travelling along edges. An example of a common tree is given in fig. 1(a). A rooted tree is a common tree where one vertex was selected. This particular vertex is called the root. The level of a vertex in a rooted tree is the number of edges that separates this vertex from the root. The root has level 0 . It is natural to draw a rooted tree by putting the root at the bottom and drawing a vertex above another one if its level is larger. The rooted tree of fig. 1)(b) was obtained by selecting as the root the lowest left vertex of fig. 1(a). The root is indicated by a white dot and a dangling line. In a rooted tree, the children of a vertex $v$ are the vertices $v^{\prime}$ that are linked to $v$ by an edge and such that the level of $v^{\prime}$ is larger than the level of $v$. A plane rooted binary tree is a rooted tree where each vertex has zero, one or two children, and each edge is oriented either to the left or to the right. If a vertex has two children, then one of the edges is oriented to the left and the other one to the right. Fig. 1(c) shows one of the plane rooted trees that can be obtained from the rooted tree of fig. 1(b). The adjective "plane" means that an edge going to the right cannot be identified with, or deformed into an edge going to the left. A plane rooted complete binary tree is a plane rooted binary tree where each vertex is "completed" by drawing leaves as follows: if a vertex has no child, draw a leaf (i.e. a line) to the left and one to the right, if a vertex has one child, then draw a leaf to the right if the child is to the left and draw it to the left if the child is to the right. Fig. 1(d) shows the plane rooted complete binary tree that is obtained from the plane rooted 


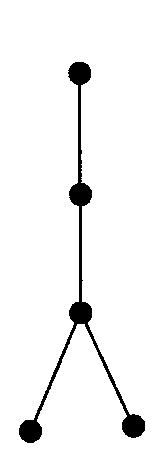

(a)

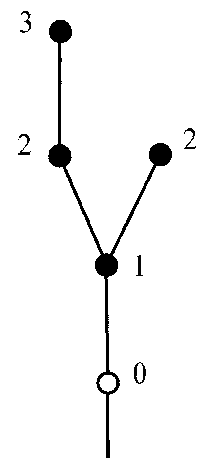

(b)

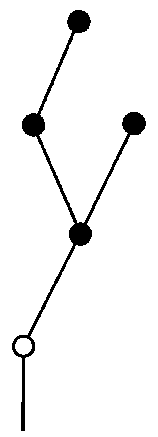

(c)

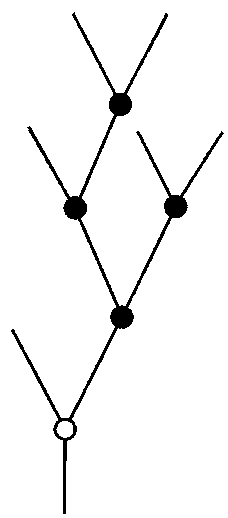

(d)

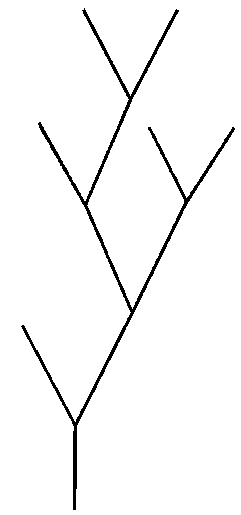

(f)

FIG. 1: Construction of plane rooted complete binary tree. (a) a common tree; (b) a rooted tree with its vertex levels; (c) a plane rooted binary tree; (d) a plane rooted complete binary tree; (f) a simplified plane rooted complete binary tree.

binary tree of fig. 1(c). In practice, the vertices are no longer necessary and they are not drawn, as in fig. I(f). If $Y_{n}$ denotes the set of plane rooted complete binary trees with $n$ vertices, we see that $Y_{1}=\{Y\}, Y_{2}=\{Y, Y\}$, $Y_{3}=\{Y, Y, Y Y, Y, Y\}$. They are much more numerous than the common trees (there is only one common tree with one, two or three vertices). For notational convenience, plane rooted complete binary trees will be simply called "trees" in the rest of the paper.

Fortunately, there exists a much simpler definition of the trees, that use a sort of building rule. We first denote the empty tree, i.e. the tree with no vertex, by $\mid$, which is a dangling line without root (a dangling line with a root and no other vertex belongs to the tree $Y$ ). Then, for any integer $n, Y_{n}$, is defined recursively by $Y_{0}:=\{\mid\}$ and, for $n>0, Y_{n}:=\left\{T_{1} \vee T_{2}: T_{1} \in Y_{k}, T_{2} \in Y_{n-k-1}, k=0, \ldots, n-1\right\}$, where $T_{1} \vee T_{2}$ is the grafting of the two trees $T_{1}$ and $T_{2}$, by which the dangling lines of $T_{1}$ and $T_{2}$ are brought together and a new root (with its own dangling line) is grown at their juncture. For example, $|\vee|=Y, \mid \vee Y=Y, Y \vee Y=Y$. Note that each tree of $Y_{n}$ has $n$ vertices (including a root) and $n+1$ leaves. The order $|T|$ of a tree $T$ is the number of its vertices.

If $C_{n}$ denotes the number of elements of $Y_{n}$, the recursive definition of $Y_{n}$ implies that $C_{0}=1$ and $C_{n}=$ $\sum_{k=0}^{n-1} C_{k} C_{n-k-1}$, so that $C_{n}=\frac{1}{n+1}\left(\begin{array}{c}2 n \\ n\end{array}\right)$ are the famous Catalan numbers. For $n=0$ to $10, C_{n}=1,1,2,5,14$, 42, 132, 429, 1430, 4862, 16796. The Catalan numbers enumerate a large number of (sometimes quite different) combinatorial objects 31 . The main practical interest of trees with respect to other combinatorial interpretations is that their recursive definition is very easy to implement.

We noticed in the previous section that, at order three, the terms $\Omega_{\sigma}$ corresponding to two specific permutations $\sigma$ can be added to give a simple result. At the general order, we shall see that the sum of $\Omega_{\sigma}$ is simple if it is carried out over permutations $\sigma$ associated with the same tree. But for this, we need to associate a tree to each permutation. The relevant map from permutations to trees belongs to "the ABC's of classical enumeration" $\underline{\underline{2}}$ and is historically one of the founding tools of modern bijective and algebraic combinatorics $13,23,33$. We describe it in the next section.

\section{From permutations to trees}

We first map any $n$-tuple $I=\left(i_{1}, \ldots, i_{n}\right)$ of distinct integers to a tree $T \in Y_{n}$. The mapping $\phi$ is defined recursively as follows: if $I=(i)$ contains a single integer, then $\phi(I):=Y$; otherwise, pick up the smallest element $i_{k}$ of $I$, then

$$
\begin{aligned}
& \phi(I):=\mid \vee \phi\left(\left(i_{2}, \ldots, i_{n}\right)\right), \text { if } k=1, \\
& \phi(I):=\phi\left(\left(i_{1}, \ldots, i_{n-1}\right)\right) \vee \mid, \text { if } k=n, \\
& \phi(I):=\phi\left(\left(i_{1}, \ldots, i_{k-1}\right)\right) \vee \phi\left(\left(i_{k+1}, \ldots, i_{n}\right)\right), \text { otherwise. }
\end{aligned}
$$


In the following, we frequently abuse notation by writing $\phi\left(i_{1}, \ldots, i_{n}\right)$ or even $\phi\left(i_{1} \ldots i_{n}\right)$ instead of $\phi\left(\left(i_{1}, \ldots, i_{n}\right)\right)$. For a permutation $\sigma \in \mathcal{S}_{n}$, the corresponding tree is $\phi(\sigma)=\phi(\sigma(1), \ldots, \sigma(n))$. For example

$$
\begin{aligned}
\phi(1) & =Y, \\
\phi(12) & =Y, \quad \phi(21)=Y, \\
\phi(123) & =Y, \quad \phi(132)=Y, \quad \phi(213)=Y \\
\phi(231) & =Y, \quad \phi(312)=Y, \quad \phi(321)=Y .
\end{aligned}
$$

Note that the two permutations (213) and (312) correspond to the same tree, and they are also the two permutations that add up to a simple sum in the calculation of $Z$ at the end of section IIB. This is not a coincidence.

To simplify the proofs, we embrace the three cases of the definition of $\phi$ into a single one as follows. We first define the concatenation product of two tuples $I=\left(i_{1}, \ldots, i_{n}\right)$ and $J=\left(j_{1}, \ldots, j_{m}\right)$ as $I \cdot J=\left(i_{1}, \ldots, i_{n}, j_{1}, \ldots, j_{m}\right)$. We extend this definition to the case of the zero-tuple $I_{0}=\emptyset$ by $I_{0} \cdot I=I \cdot I_{0}=I$. Then, for any $n$-tuple $I$ of distinct integers, we define $\phi(I)$ by $\phi(I)=\mid$ if $n=0$ and $\phi(I)=\phi\left(I_{1}\right) \vee \phi\left(I_{2}\right)$ otherwise, where $I_{1}$ and $I_{2}$ are determined by $I=I_{1} \cdot(\min I) \cdot I_{2}$. Note that $I_{1}$ or $I_{2}$ can be the zero-tuple.

We first prove an easy lemma.

Lemma 2 If the elements of the two $n$-tuples $I=\left(i_{1}, \ldots, i_{n}\right)$ and $J=\left(j_{1}, \ldots, j_{n}\right)$ of distinct integers have the same ordering (i.e. if $i_{k}<i_{l}$ if and only if $j_{k}<j_{l}$ for all $k$ and $l$ in $\left.\{1, \ldots, n\}\right)$, then $\phi(I)=\phi(J)$.

Proof. The proof is by induction. If $n=0$, then $I=J=\emptyset$ and $\phi(I)=\phi(J)=\mid$. Assume that the property is true for $k$-tuples of distinct integers up to $k=n-1$ and take two $n$-tuples $I$ and $J$ having the same ordering. Then, the minimum element of both is at the same position $k$ (i.e. $\min I=i_{k}$ and $\left.\min J=j_{k}\right)$ and $I=I_{1} \cdot\left(i_{k}\right) \cdot I_{2}$, $J=J_{1} \cdot\left(j_{k}\right) \cdot J_{2}$, where $I_{1}$ and $J_{1}\left(I_{2}\right.$ and $J_{2}$, respectively) are two $(k-1)$-tuples $((n-k)$-tuples, respectively) of distinct integers have the same ordering. By the recursion hypothesis, we have $\phi\left(I_{1}\right)=\phi\left(J_{1}\right)$ and $\phi\left(I_{2}\right)=\phi\left(J_{2}\right)$ and the definition of $\phi$ gives us $\phi(I)=\phi\left(I_{1}\right) \vee \phi\left(I_{2}\right)=\phi\left(J_{1}\right) \vee \phi\left(J_{2}\right)=\phi(J)$.

As a useful particular case, we consider the situation where $J$ describes the ordering of the elements of $I$ : we order the elements of $I=\left(i_{1}, \ldots, i_{n}\right)$ increasingly as $i_{k_{1}}<\cdots<i_{k_{n}}$. Then $j_{l}$ is the position of $i_{l}$ in this ordering. More formally, $J:=\left(\tau^{-1}(1), \ldots, \tau^{-1}(n)\right)$, where $\tau$ is the permutation $\left(k_{1}, \ldots, k_{n}\right)$. The $n$-tuple $J$ is called the standardization of $I$ and it is denoted by $\operatorname{st}(I)$. If we take the example of $I=(5,8,2)$, the position of 5,8 and 2 in the ordering $2<5<8$ is 2,3 and 1 , respectively. Thus, st $(5,8,2)=(2,3,1)$. By construction, $I$ and $s t(I)$ have the same ordering and $\phi(I)=\phi(s t(I))$. We extend the standardization to the case of $I=\emptyset$ by $\operatorname{st}(\emptyset)=\emptyset$.

\section{From trees to permutations}

Conversely, we shall need to know the permutations corresponding to a given tree: $S_{T}:=\left\{\sigma \in \mathcal{S}_{|T|}: \phi(\sigma)=T\right\}$ (we extend this definition to the case of $T=\mid$ by defining the zero-element permutation group $\mathcal{S}_{0}:=\{\emptyset\}$ ). The solution of this problem is given by

Lemma 3 If $T=T_{1} \vee T_{2}$, where $\left|T_{1}\right|=n$ and $\left|T_{2}\right|=m$ ( $n$ or $m$ can be zero), all the permutations of $S_{T}$ have the form $I=I_{1} \cdot(1) \cdot I_{2}$, where $I_{1}$ is a subset of $n$ elements of $\{2, \ldots, n+m+1\}$ ordered according to a permutation $\alpha$ of $S_{T_{1}}$ (i.e. $\operatorname{st}\left(I_{1}\right)=\alpha$ ) and $I_{2}$ is the complement of $I_{1}$ in $\{2, \ldots, n+m+1\}$, ordered according to a permutation $\beta$ of $S_{T_{2}}$ (i.e. $\operatorname{st}\left(I_{2}\right)=\beta$ ).

Proof. The proof is given in refs. 29 and 30 , but we can sketch it here for completeness. The simplest examples are $S_{T}=\{\emptyset\}$ for $T=\mid$ and $S_{T}=\{(1)\}$ for $T=Y$. Now take $T=T_{1} \vee T_{2}$ as in the lemma. By the definition of $\phi$, the minimum of the tuple $I=(\sigma(1), \ldots, \sigma(n+m+1))$ is $\sigma(n+1)=1$ and $I=I_{1} \cdot(1) \cdot I_{2}$, where $\phi\left(I_{1}\right)=T_{1}$ and $\phi\left(I_{2}\right)=T_{2}$. We saw in the previous section that $\phi\left(I_{1}\right)=\phi\left(s t\left(I_{1}\right)\right)$. By definition $\operatorname{st}\left(I_{1}\right)$ is a permutation of $\mathcal{S}_{n}$. Therefore, st $\left(I_{1}\right)$ belongs to $S_{T_{1}}$ and, similarly, st $\left(I_{2}\right)$ belongs to $S_{T_{2}}$. It is now enough to check that each element of $S_{T}$ is obtained exactly once by running the construction over all orderings and all permutations of $T_{1}$ and $T_{2}$.

This lemma allows us to recursively determine the number of elements of $S_{T}$, denoted by $\left|S_{T}\right|$, by $\left|S_{T}\right|=1$ for $T=\mid$ and $T=Y$ and, for $T=T_{1} \vee T_{2}$,

$$
\left|S_{T}\right|=\left(\begin{array}{c}
|T|-1 \\
\left|T_{1}\right|
\end{array}\right)\left|S_{T_{1}}\right|\left|S_{T_{2}}\right| .
$$


See ref. 29 for an alternative approach.

Example: Consider the tree $T=T_{1} \vee T_{2}$, with $T_{1}=Y$ and $T_{2}=Y$, so that $n=1$ and $m=3$. $S_{T_{1}}$ contains the single permutation $\alpha=(1)$ and, according to the examples given in the previous section, the two permutations of $S_{T_{2}}$ are $\beta_{1}=(213)$ and $\beta_{2}=(312)$. We choose the permutations $\alpha$ and $\beta_{1}$, we pick up $n=1$ element (for example 3 ) in the set $J=\{2,3,4,5\}$, so that $I_{1}=(3)$ and we order the remaining elements $\{2,4,5\}$ according to $\beta_{1}$, so that $I_{2}=(4,2,5)$. This gives us $\sigma=(31425)$. If we pick up the other elements of $J$ to build $I_{1}$ we obtain (21435), (41325) and (51324). We add the elements obtained by choosing $\beta_{2}$ and we obtain eight permutations:

$$
S_{T}=\{(21435),(21534),(31425),(31524),(41325),(41523),(51324),(51423)\} .
$$

We can check that eq. (7) holds and that $\left|S_{T}\right|=8$.

\section{Recursion formula}

The permutations corresponding to a tree can be used to make a partial summation of the terms of the Picard-Dyson expansion.

Definition 4 For any tree $T$, we define $\Omega_{T}\left(t, t_{0}\right)$ by $\Omega_{T}\left(t, t_{0}\right)=P$ if $T=\mid$ and

$$
\Omega_{T}\left(t, t_{0}\right):=\sum_{\sigma \in S_{T}} \int_{t_{0}}^{t} \mathrm{~d} t_{1} \int_{t_{0}}^{t_{1}} \mathrm{~d} t_{2} \ldots \int_{t_{0}}^{t_{n-1}} \mathrm{~d} t_{n} X\left(t_{\sigma(1)}\right) \ldots X\left(t_{\sigma(n)}\right),
$$

otherwise, where $n=|T|$.

With this notation we have obviously $\Omega\left(t, t_{0}\right)=\sum_{T} \Omega_{T}\left(t, t_{0}\right)=\sum_{\sigma} \Omega_{\sigma}\left(t, t_{0}\right)$, with the notation $\Omega_{\sigma}\left(t, t_{0}\right):=$ $\int_{t_{0}}^{t} \mathrm{~d} t_{1} \int_{t_{0}}^{t_{1}} \mathrm{~d} t_{2} \ldots \int_{t_{0}-1}^{t_{n-1}} \mathrm{~d} t_{n} X\left(t_{\sigma(1)}\right) \ldots X\left(t_{\sigma(n)}\right)$ and where $\sigma$ runs over all permutations (of all orders). The term of order 0 of this series is $\Omega_{\mid}=P$ and the term of order one is

$$
\Omega_{T}\left(t, t_{0}\right)=-\imath \int_{t_{0}}^{t} \mathrm{~d} s Q H(s) P
$$

for $T=Y$. The other terms enjoy a remarkably simple recurrence relation:

Theorem 5 If $|T|>1$, then $\Omega_{T}\left(t, t_{0}\right)$ can be expressed recursively by

$$
\begin{aligned}
& \Omega_{T}\left(t, t_{0}\right)=-\imath \int_{t_{0}}^{t} \mathrm{~d} s Q H(s) \Omega_{T_{2}}\left(s, t_{0}\right), \text { if } T=\mid \vee T_{2}, \\
& \Omega_{T}\left(t, t_{0}\right)=\imath \int_{t_{0}}^{t} \mathrm{~d} s \Omega_{T_{1}}\left(s, t_{0}\right) H(s) P, \text { if } T=T_{1} \vee \mid, \\
& \Omega_{T}\left(t, t_{0}\right)=\imath \int_{t_{0}}^{t} \mathrm{~d} s \Omega_{T_{1}}\left(s, t_{0}\right) H(s) \Omega_{T_{2}}\left(s, t_{0}\right), \text { if } T=T_{1} \vee T_{2},
\end{aligned}
$$

where $T_{1} \neq \mid$ and $T_{2} \neq 1$.

Note that a similar recursive expression was conjectured by Olszewski for the nondegenerate Rayleigh-Schrödinger expansion 27 .

Proof. Let us prove the theorem recursively. Consider an arbitrary $T=T_{1} \vee T_{2},|T|>1$, and assume the formulas to hold for all the trees $T^{\prime}$ with $\left|T^{\prime}\right|<|T|$. Consider for example the case where $T_{1} \neq \mid$ and $T_{2} \neq \mid$ (the other cases are even simpler). We define:

$$
A_{T}:=\imath \int_{t_{0}}^{t} \mathrm{~d} s \Omega_{T_{1}}\left(s, t_{0}\right) H(s) \Omega_{T_{2}}\left(s, t_{0}\right)=\sum_{\alpha \in S_{T_{1}}, \beta \in S_{T_{2}}} \imath \int_{t_{0}}^{t} \mathrm{~d} s \Omega_{\alpha}\left(s, t_{0}\right) H(s) \Omega_{\beta}\left(s, t_{0}\right) .
$$

This first important point is that, for a given tree $T$, all the permutations $\sigma \in S_{T}$ have the same descent set. This is a well-known fact ${ }^{23,30}$ that can be deduced from the characterization of $S_{T}$ at the end of section $\amalg$ C. As a consequence, 
the sequence of operators $P, Q$ and $H$ is the same for all $\alpha$ and $\beta$ in $A_{T}$, and only the order of the arguments $t_{i}$ varies. Therefore, the conditions of lemma 16 (see appendix A) are satisfied and we get:

$$
A_{T}=\sum_{\gamma} \Omega_{\gamma}\left(t, t_{0}\right)
$$

where $\gamma$ runs over the permutations such that $\gamma\left(\left|T_{1}\right|+1\right)=1$, st $\left(\gamma(1), \ldots, \gamma\left(\left|T_{1}\right|\right)\right) \in S_{T_{1}}, \operatorname{st}\left(\gamma\left(\left|T_{1}\right|+2\right), \ldots, \gamma\left(\left|T_{1}\right|+\right.\right.$ $\left.\left.\left|T_{2}\right|+1\right)\right) \in S_{T_{2}}$. The set of permutations $\gamma$ satisfying these equations is precisely $S_{T}$, so that, finally: $A_{T}=\Omega_{T}\left(t, t_{0}\right)$. This concludes the proof of the theorem.

\section{E. Remarks}

\section{Nonlinear integral equation}

If we denote $\chi\left(t, t_{0}\right)=\Omega\left(t, t_{0}\right)-P$, then the recurrence relations add up to

$$
\imath \chi\left(t, t_{0}\right)=\int_{t_{0}}^{t} \mathrm{~d} s Q H(s) P+\int_{t_{0}}^{t} \mathrm{~d} s Q H(s) \chi\left(s, t_{0}\right)-\int_{t_{0}}^{t} \mathrm{~d} s \chi\left(s, t_{0}\right) H(s) P-\int_{t_{0}}^{t} \mathrm{~d} s \chi\left(s, t_{0}\right) H(s) \chi\left(s, t_{0}\right) .
$$

The derivative of this equation with respect to $t$ was obtained in a different way by Jolicard $\underline{3}$.

\section{Permutations, trees and descents}

We saw that, for a given tree $T$, all permutations of $S_{T}$ have the same descent set. We can now give more details 30,34 . The relation between the trees and the sequences of operators $P$ and $Q$ in eq. (3) is very simple. Consider the sequence of leaves from left to right. Each leaf pointing to the right corresponds to a $P$, each leaf pointing to the left correspond

to a $Q$. For example, the tree $Y$ corresponds to the sequence $Q Q P P$. From the combinatorial point of view, this description emphasizes the existence of a relationship between trees and descent sets (or, equivalently, hypercubes), see e.g. refs. 23,34 and our Appendix.

\section{ADIABATIC SWITCHING}

Morita's formula is most often applied to an interaction Hamiltonian $H^{\epsilon}(t):=\mathrm{e}^{-\epsilon|t|} \mathrm{e}^{\imath H_{0} t} V \mathrm{e}^{-\imath H_{0} t}$. We write $E_{0}, \ldots, E_{n}, \ldots$ and $\Phi_{0}, \ldots, \Phi_{n}, \ldots$ for the eigenvalues of $H_{0}$ and for an orthogonal basis of corresponding eigenstates. We assume that the spectrum is discrete and that the eigenvalues are ordered by (weakly) increasing order. The ground state may be degenerate $\left(E_{0}=E_{1}=\ldots=E_{k}\right.$ for a given $\left.k\right)$. The model space $M$ (see the Introduction) is the vector space generated by the lowest $N$ eigenstates of $H_{0}$ (with $N \geq k$ ). We assume that the energies of the eigenstates of $M$ are separated by a finite gap from the energies of the eigenstates that do not belong to $M$. The projector $P$ is the projector onto the model space $M$. Following the notation of the Introduction, the energies of the eigenstates that belong (resp. do not belong) to $M$ are denoted by $E_{i}^{P}$ (resp. $E_{i}^{Q}$ ).

In this section, we prove the convergence of each term of the perturbation expansion of the wave operator when $\epsilon \rightarrow 0$. For notational convenience, we assume that $t \leq 0$. We first give a nonperturbative proof of this convergence. Then, we expand in series and we consider the different cases of the previous sections.

\section{A. Nonperturbative proof}

The nonperturbative proof is important in this context because its range of validity is wider than the series expansion (no convergence criterion for the series is required). Moreover, the proof that the wave operator indeed leads to an effective Hamiltonian is much easier to give in the nonperturbative setting.

The first condition required in the nonperturbative setting is that the perturbation $V$ must be relatively bounded with respect to $H_{0}$ with a bound strictly smaller than 1 . This condition is satisfied for the Hamiltonian describing

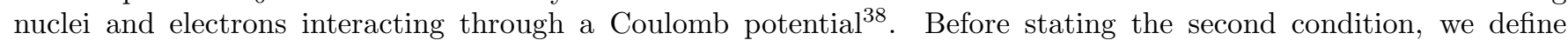
the time independent Hamiltonian $h(\lambda)=H_{0}+\lambda V$, its eigenvalues $E_{j}(\lambda)$ and its eigenprojectors $P_{j}(\lambda)$, such that 
$h(\lambda) P_{j}(\lambda)=E_{j}(\lambda) P_{j}(\lambda)$. The second condition is that the eigenvalues $E_{j}(\lambda)$ coming from the eigenstates of the model space (i.e. such that $\left.P_{j}(0) P=P_{j}(0)\right)$ are separated by a finite gap from the rest of the spectrum. According to Kato $\frac{38}{3}$, the eigenvalues and eigenprojectors can be chosen analytic in $\lambda$. Then, a recent version of the adiabatic theorem ${ }^{39,40}$ shows that there exists a unitary operator $A$, independent of $\epsilon$, such that

$$
\lim _{\epsilon \rightarrow 0}\left\|U_{\epsilon}(0,-\infty) P_{j}(0)-\mathrm{e}^{\imath \theta_{j} / \epsilon} A P_{j}(0)\right\|=0,
$$

where $U_{\epsilon}\left(t, t_{0}\right)$ is the evolution operator for the Hamiltonian $H^{\epsilon}(t)=\mathrm{e}^{-\epsilon|t|} \mathrm{e}^{\imath H_{0} t} V \mathrm{e}^{-\imath H_{0} t}$ and

$$
\theta_{j}=\int_{0}^{1} \frac{E_{j}(0)-E_{j}(\lambda)}{\lambda} \mathrm{d} \lambda .
$$

In other words, the singularity of $U_{\epsilon}(0,-\infty) P_{j}(0)$ is entirely described by the factor $\mathrm{e}^{\imath \theta_{j} / \epsilon}$. The operator $A$ satisfies the intertwining property $A P_{j}(0)=P_{j}(1) A$. To connect this result with the case that we are considering in this paper, we have to choose a model space $M$ that satisfies the following condition: there is a set $I$ of indices $j$ such that $P=\sum_{j \in I} P_{j}(0)$, where $P$ is the projector onto $M$.

This enables us to give a more precise condition for the invertibility of $P U_{\epsilon}(0,-\infty) P$. The adiabatic theorem shows that, for small enough $\epsilon, P U_{\epsilon}(0,-\infty) P$ is invertible if and only if $P A P$ is invertible. If we rewrite $P A P=$ $\sum_{j} P A P_{j}(0)=\sum_{j} P P_{j}(1) A$, the unitarity of $A$ implies that $P A P$ is invertible iff the kernel of $\sum_{j} P P_{j}(1)$ is trivial. We recover the well-known invertibility condition $\frac{18}{}$ that no state of the model space should be orthogonal to the vector space spanned by all the eigenstates of $H$ with energy $E_{j}(1)$, where $j$ runs over $I$. Note that, when the condition of invertibility is not satisfied, it can be recovered by adding the perturbation step by step $\underline{39}$.

Then, we have

Theorem 6 With the given conditions, the wave operator

$$
\bar{\Omega}:=\lim _{\epsilon \rightarrow 0} U_{\epsilon}(0,-\infty) P\left(P U_{\epsilon}(0,-\infty) P\right)^{-1}
$$

is well defined. Moreover, there are states $\left|\tilde{\varphi}_{j}\right\rangle$ in the model space such that $\bar{\Omega}\left|\tilde{\varphi}_{j}\right\rangle$ is an eigenstate of $H$ with eigenvalue $E_{j}(1)$ and the effective Hamiltonian $H_{\mathrm{eff}}:=P H \bar{\Omega}$ satisfies $H_{\mathrm{eff}}\left|\tilde{\varphi}_{j}\right\rangle=E_{j}(1)\left|\tilde{\varphi}_{j}\right\rangle$.

Proof. We first define $A_{j k}=P_{j}(0) A P_{k}(0)$, for $j$ and $k$ in $I$. Then an inverse $B$ of $P A P$ is defined by $\sum_{k \in I} A_{j k} B_{k l}=$ $\delta_{j l} P_{j}(0)$ where $B_{j k}=P_{j}(0) B P_{k}(0)$. Then, $\left(P U_{\epsilon}(0,-\infty) P\right)^{-1} \simeq \sum_{j k} \mathrm{e}^{-\imath \theta_{j} / \epsilon} B_{j k}$ and

$$
\Omega_{\epsilon}(0,-\infty):=U_{\epsilon}(0,-\infty) P\left(P U_{\epsilon}(0,-\infty) P\right)^{-1} \simeq \sum_{j k} A P_{j}(0) B_{j k} .
$$

Since the right hand side does not depend on $\epsilon$, then $\Omega_{\epsilon}(0,-\infty)$ has no singularity at $\epsilon=0$ and

$$
\bar{\Omega}=\lim _{\epsilon \rightarrow 0} \Omega_{\epsilon}(0,-\infty)=\sum_{j k} A P_{j}(0) B_{j k} .
$$

This proves the existence of the wave operator. To prove the existence of the states $\left|\tilde{\varphi}_{j}\right\rangle$ of the theorem, define $\left|\tilde{\varphi}_{j}\right\rangle=P A\left|\varphi_{j}\right\rangle$, where $\left|\varphi_{j}\right\rangle$ is an eigenstate of $P_{j}(0): P_{j}(0)\left|\varphi_{j}\right\rangle=\left|\varphi_{j}\right\rangle$. Indeed, we have

$$
\bar{\Omega}\left|\tilde{\varphi}_{j}\right\rangle=\bar{\Omega} P A P_{j}(0)\left|\varphi_{j}\right\rangle=\sum_{k m} A P_{k}(0) B_{k m} A_{m j}\left|\varphi_{j}\right\rangle=A P_{j}(0)\left|\varphi_{j}\right\rangle=P_{j}(1) A\left|\varphi_{j}\right\rangle,
$$

where we used the intertwining property in the last equation. We can now check that $\bar{\Omega}\left|\tilde{\varphi}_{j}\right\rangle$ is an eigenstate of $H$ with eigenvalue $E_{j}(1)$.

$$
H \bar{\Omega}\left|\tilde{\varphi}_{j}\right\rangle=h(1) P_{j}(1) A\left|\phi_{j}\right\rangle=E_{j}(1) P_{j}(1) A\left|\phi_{j}\right\rangle=E_{j}(1) \bar{\Omega}\left|\tilde{\varphi}_{j}\right\rangle .
$$

Finally, by multiplying eq. (9) by $P$ from the left, we obtain

$$
H_{\mathrm{eff}}\left|\tilde{\varphi}_{j}\right\rangle=E_{j}(1) P \bar{\Omega}\left|\tilde{\varphi}_{j}\right\rangle=E_{j}(1)\left|\tilde{\varphi}_{j}\right\rangle
$$

because $P \bar{\Omega}=P$ and $P\left|\tilde{\varphi}_{j}\right\rangle=\left|\tilde{\varphi}_{j}\right\rangle$.

Thus, the eigenvalues of $H_{\text {eff }}$ are eigenvalues of the full Hamiltonian $H$. This is exactly what is expected from an effective Hamiltonian. In practice, the operator $A$ is not known and the states $\left|\tilde{\varphi}_{j}\right\rangle$ are obtained by diagonalizing $H_{\text {eff }}$. 


\section{B. Series expansion}

We consider again the series expansion in terms of permutations. A straightforward calculation 41,42 of the PicardDyson series gives us

$$
U_{\epsilon}(0,-\infty)\left|\Phi_{0}\right\rangle=\left|\Phi_{0}\right\rangle+\sum_{n=1}^{\infty} \sum_{i_{1} \ldots i_{n}} \frac{\left|\Phi_{i_{1}}\right\rangle\left\langle\Phi_{i_{1}}|V| \Phi_{i_{2}}\right\rangle \ldots\left\langle\Phi_{i_{n-1}}|V| \Phi_{i_{n}}\right\rangle\left\langle\Phi_{i_{n}}|V| \Phi_{0}\right\rangle}{\left(E_{0}-E_{i_{1}}+n \imath \epsilon\right)\left(E_{0}-E_{i_{2}}+(n-1) \imath \epsilon\right) \ldots\left(E_{0}-E_{i_{n}}+\imath \epsilon\right)}
$$

where we used the completeness relation $1=\sum_{i}\left|\Phi_{i}\right\rangle\left\langle\Phi_{i}\right|$. This expression clearly shows that the terms of the expansion (and the evolution operator) are divergent as $\epsilon \rightarrow 0$ when any $E_{i_{k}}$ is equal to $E_{0}$.

For $\sigma \in \mathcal{S}_{n}$, we set $\Omega_{\sigma}(t):=\Omega_{\sigma}(t,-\infty)$. We then have

$$
\begin{aligned}
\Omega_{\sigma}(t)= & (-\imath)^{n} \int_{-\infty}^{t} \mathrm{~d} t_{1} \int_{-\infty}^{t_{1}} \mathrm{~d} t_{2} \ldots \int_{-\infty}^{t_{n-1}} \mathrm{~d} t_{n} Q \mathrm{e}^{\left(\epsilon+\imath H_{0}\right) t_{\sigma(1)}} V \mathrm{e}^{-\imath H_{0} t_{\sigma(1)}} R_{\sigma}^{1} \\
& \mathrm{e}^{\left(\epsilon+\imath H_{0}\right) t_{\sigma(2)}} V \mathrm{e}^{-\imath H_{0} t_{\sigma(2)}} R_{\sigma}^{2} \ldots R_{\sigma}^{n-1} \mathrm{e}^{\left(\epsilon+\imath H_{0}\right) t_{\sigma(n)}} V \mathrm{e}^{-\imath H_{0} t_{\sigma(n)}} P,
\end{aligned}
$$

where $R_{\sigma}^{k}:=Q$ if $\sigma(k+1)>\sigma(k)$ and $R_{\sigma}^{k}:=-P$ if $\sigma(k+1)<\sigma(k)$. We replace $R_{\sigma}^{k}$ by $\pm \sum_{\alpha_{k+1}}\left|\alpha_{k+1}\right\rangle\left\langle\alpha_{k+1}\right|$ where, if $R_{\sigma}^{k}=Q$, then $\pm=+$ and the sum is over the image of $Q$, and if $R_{\sigma}^{k}=-P$, then $\pm=-$ and the sum is over the image of $P$. Thus

$$
\begin{aligned}
\Omega_{\sigma}(t)= & (-\imath)^{n}(-1)^{d} \int_{-\infty}^{t} \mathrm{~d} t_{1} \int_{-\infty}^{t_{1}} \mathrm{~d} t_{2} \ldots \int_{-\infty}^{t_{n-1}} \mathrm{~d} t_{n} \mathrm{e}^{\left(\epsilon+\imath F_{1}-\imath F_{2}\right) t_{\sigma(1)}} \mathrm{e}^{\left(\epsilon+\imath F_{2}-\imath F_{3}\right) t_{\sigma(2)} \ldots \mathrm{e}^{\left(\epsilon+\imath F_{n}-\imath F_{n+1}\right) t_{\sigma(n)}}} \\
& \sum_{\alpha_{1} \ldots \alpha_{n+1}}\left|\alpha_{1}\right\rangle\left\langle\alpha_{1}|V| \alpha_{2}\right\rangle \ldots\left\langle\alpha_{n}|V| \alpha_{n+1}\right\rangle\left\langle\alpha_{n+1}\right|
\end{aligned}
$$

where $d$ is the number of elements of the descent set of $\sigma, F_{i}$ is the energy of $\alpha_{i}$ and where the sum over $\alpha_{1}$ is over the image of $Q$, the sum over $\alpha_{n+1}$ is over the image of $P$ and the sum over $\alpha_{k}$ for $1<k<n+1$ is over the image of $Q$ if $\sigma(k)>\sigma(k-1)$ and over the image of $P$ otherwise. Consider now the time integral

$$
\begin{aligned}
f_{\sigma}(t) & :=\int_{-\infty}^{t} \mathrm{~d} t_{1} \int_{-\infty}^{t_{1}} \mathrm{~d} t_{2} \ldots \int_{-\infty}^{t_{n-1}} \mathrm{~d} t_{n} \mathrm{e}^{\left(\epsilon+\imath F_{1}-\imath F_{2}\right) t_{\sigma(1)}} \mathrm{e}^{\left(\epsilon+\imath F_{2}-\imath F_{3}\right) t_{\sigma(2)}} \ldots \mathrm{e}^{\left.\left(\epsilon+\imath F_{n}-\imath F_{n+1}\right) t_{\sigma(n)}\right)} \\
& =\int_{-\infty}^{t} \mathrm{~d} s_{\tau(1)} \int_{-\infty}^{s_{\tau(1)}} \mathrm{d} s_{\tau(2)} \cdots \int_{-\infty}^{s_{\tau(n-1)}} \mathrm{d} s_{\tau(n)} \mathrm{e}^{\left(\epsilon+\imath F_{1}-\imath F_{2}\right) s_{1}} \mathrm{e}^{\left(\epsilon+\imath F_{2}-\imath F_{3}\right) s_{2}} \ldots \mathrm{e}^{\left(\epsilon+\imath F_{n}-\imath F_{n+1}\right) s_{n}}
\end{aligned}
$$

where $\tau=\sigma^{-1}$. The integral over $s_{\tau(n)}$ is

$$
\int_{-\infty}^{s_{\tau(n-1)}} \mathrm{d} s_{\tau(n)} \mathrm{e}^{\left(\epsilon+\imath F_{\tau(n)}-\imath F_{\tau(n)+1}\right) s_{\tau(n)}}=\frac{\mathrm{e}^{\left(\epsilon+\imath F_{\tau(n)}-\imath F_{\tau(n)+1}\right) s_{\tau(n-1)}}}{\left(\epsilon+\imath F_{\tau(n)}-\imath F_{\tau(n)+1}\right)} .
$$

The integrand of the integral over $s_{\tau(n-1)}$ becomes

$$
\frac{\mathrm{e}^{\left(2 \epsilon+\imath\left(F_{\tau(n)}+F_{\tau(n-1)}-F_{\tau(n)+1}-F_{\tau(n-1)+1}\right) s_{\tau(n-1)}\right.}}{\left(\epsilon+\imath F_{\tau(n)}-\imath F_{\tau(n)+1}\right)} .
$$

A straightforward recursive argument shows that

$$
f_{\sigma}(t)=\frac{\mathrm{e}^{X_{\sigma}(n) t}}{X_{\sigma}(1) \ldots X_{\sigma}(n)}
$$

where

$$
X_{\sigma}(k):=k \epsilon+\imath\left(F_{\sigma^{-1}(n)}+\cdots+F_{\sigma^{-1}(n-k+1)}-F_{\sigma^{-1}(n)+1}-\cdots-F_{\sigma^{-1}(n-k+1)+1}\right) .
$$

Therefore,

$$
\Omega_{\sigma}(t)=\sum_{\alpha_{1} \ldots \alpha_{n+1}} \frac{(-\imath)^{n}(-1)^{d} \mathrm{e}^{X_{\sigma}(n) t}}{X_{\sigma}(1) \ldots X_{\sigma}(n)}\left|\alpha_{1}\right\rangle\left\langle\alpha_{1}|V| \alpha_{2}\right\rangle \ldots\left\langle\alpha_{n}|V| \alpha_{n+1}\right\rangle\left\langle\alpha_{n+1}\right|
$$




\section{Examples}

A few examples of $\Omega_{\sigma}(0)$ are

$$
\begin{aligned}
\Omega_{(1)}(0) & =(-\imath) \sum_{\Phi_{i} \Phi_{j}} \frac{\left|\Phi_{i}\right\rangle\left\langle\Phi_{i}|V| \Phi_{j}\right\rangle\left\langle\Phi_{j}\right|}{\epsilon+\imath\left(E_{i}^{Q}-E_{j}^{P}\right)} \\
\Omega_{(12)}(0) & =(-\imath)^{2} \sum_{\Phi_{i} \Phi_{j} \Phi_{k}} \frac{\left|\Phi_{i}\right\rangle\left\langle\Phi_{i}|V| \Phi_{j}\right\rangle\left\langle\Phi_{j}|V| \Phi_{k}\right\rangle\left\langle\Phi_{k}\right|}{\left(\epsilon+\imath\left(E_{j}^{Q}-E_{k}^{P}\right)\right)\left(2 \epsilon+\imath\left(E_{i}^{Q}-E_{k}^{P}\right)\right)}, \\
\Omega_{(21)}(0) & =-(-\imath)^{2} \sum_{\Phi_{i} \Phi_{j} \Phi_{k}} \frac{\left|\Phi_{i}\right\rangle\left\langle\Phi_{i}|V| \Phi_{j}\right\rangle\left\langle\Phi_{j}|V| \Phi_{k}\right\rangle\left\langle\Phi_{k}\right|}{\left(\epsilon+\imath\left(E_{i}^{Q}-E_{j}^{P}\right)\right)\left(2 \epsilon+\imath\left(E_{i}^{Q}-E_{k}^{P}\right)\right)} .
\end{aligned}
$$

Finally we consider two examples that will prove useful:

$$
\begin{aligned}
& \Omega_{(213)}(0)=-(-\imath)^{3} \sum_{\Phi_{i} \Phi_{j} \Phi_{k} \Phi_{l}} \frac{\left|\Phi_{i}\right\rangle\left\langle\Phi_{i}|V| \Phi_{j}\right\rangle\left\langle\Phi_{j}|V| \Phi_{k}\right\rangle\left\langle\Phi_{k}|V| \Phi_{l}\right\rangle\left\langle\Phi_{l}\right|}{\left(\epsilon+\imath\left(E_{k}^{Q}-E_{l}^{P}\right)\right)\left(2 \epsilon+\imath\left(E_{k}^{Q}+E_{i}^{Q}-E_{l}^{P}-E_{j}^{P}\right)\right)\left(3 \epsilon+\imath\left(E_{i}^{Q}-E_{l}^{P}\right)\right)}, \\
& \Omega_{(312)}(0)=-(-\imath)^{3} \sum_{\Phi_{i} \Phi_{j} \Phi_{k} \Phi_{l}} \frac{\left|\Phi_{i}\right\rangle\left\langle\Phi_{i}|V| \Phi_{j}\right\rangle\left\langle\Phi_{j}|V| \Phi_{k}\right\rangle\left\langle\Phi_{k}|V| \Phi_{l}\right\rangle\left\langle\Phi_{l}\right|}{\left(\epsilon+\imath\left(E_{i}^{Q}-E_{j}^{P}\right)\right)\left(2 \epsilon+\imath\left(E_{k}^{Q}+E_{i}^{Q}-E_{l}^{P}-E_{j}^{P}\right)\right)\left(3 \epsilon+\imath\left(E_{i}^{Q}-E_{l}^{P}\right)\right)} .
\end{aligned}
$$

By adding these two terms, we obtain a denominator involving only the difference of two energies.

$$
\Omega_{(213)}(0)+\Omega_{(312)}(0)=-(-\imath)^{3} \sum_{\Phi_{i} \Phi_{j} \Phi_{k} \Phi_{l}} \frac{\left|\Phi_{i}\right\rangle\left\langle\Phi_{i}|V| \Phi_{j}\right\rangle\left\langle\Phi_{j}|V| \Phi_{k}\right\rangle\left\langle\Phi_{k}|V| \Phi_{l}\right\rangle\left\langle\Phi_{l}\right|}{\left(\epsilon+\imath\left(E_{i}^{Q}-E_{j}^{P}\right)\right)\left(\epsilon+\imath\left(E_{k}^{Q}-E_{l}^{P}\right)\right)\left(3 \epsilon+\imath\left(E_{i}^{Q}-E_{l}^{P}\right)\right)} .
$$

Note that the sum is simpler than either $\Omega_{(213)}(0)$ or $\Omega_{(312)}(0)$. This is a general statement and the simplification becomes spectacular at higher orders. For the example of $n=7$, there is a single tree $T$ which is the sum of 80 permutations, and the denominator of $\Omega_{T}$ is simpler than the denominator of $\Omega_{\sigma}$ for any of the 80 permutations $\sigma$ of $S_{T}$. This will be proved in section IV Note also that, if we assume that the states in the image of $P$ (i.e. the model space) are separated from the states in the image of $Q$ by a finite gap $\delta$, so that $E_{i}^{Q}-E_{j}^{P} \geq \delta$, then the denominators of all the examples are non-zero when $\epsilon \rightarrow 0$. In other words, the limit $\lim _{\epsilon \rightarrow 0} \Omega_{\sigma}(0)$ exists for all the examples. In the next section, we show that this result is true for all permutations $\sigma$.

\section{Convergence of $\Omega_{\sigma}(t)$}

Definition (11) is convenient for a computer implementation but it does not make it clear that $X_{\sigma}(k)$ is nonzero if $\epsilon=0$. For that purpose, we need an alternative expression for $X_{\sigma}(k)$, which is essentially a corrected version of the graphical rule given by Michels and Suttorp ${ }^{19}$. We first extend any permutation $\sigma \in \mathcal{S}_{n}$ to the sequence of $n+2$ integers $\bar{\sigma}=(\bar{\sigma}(1), \ldots, \bar{\sigma}(n+2))=(0, \sigma(1), \ldots, \sigma(n), 0)$. Then, for $k \in\{1, \ldots, n\}$, we define the two sets

$$
\begin{aligned}
& S_{\sigma}^{<}(k):=\{i \mid 1 \leq i \leq n+1 \text { and } \bar{\sigma}(i)<k \leq \bar{\sigma}(i+1)\}, \\
& S_{\sigma}^{>}(k):=\{i \mid 1 \leq i \leq n+1 \text { and } \bar{\sigma}(i) \geq k>\bar{\sigma}(i+1)\} .
\end{aligned}
$$

For example, if $\sigma=(41325)$, then $S_{\sigma}^{<}(1)=\{1\}, S_{\sigma}^{<}(2)=\{1,3\}, S_{\sigma}^{<}(3)=\{1,3,5\}, S_{\sigma}^{<}(4)=\{1,5\}, S_{\sigma}^{<}(5)=\{5\}$ and $S_{\sigma}^{>}(1)=\{6\}, S_{\sigma}^{>}(2)=\{2,6\}, S_{\sigma}^{>}(3)=\{2,4,6\}, S_{\sigma}^{>}(4)=\{2,6\}, S_{\sigma}^{>}(5)=\{6\}$. The graphical meaning of these sets is illustrated in Figure 2, Notice that the vertical axis is oriented downwards in order to reflect the time-ordering in the integrals $S_{\sigma}(t)$.

Lemma 7 (i) $S_{\sigma}^{<}(k)$ cannot be empty and (ii) $S_{\sigma}^{<}(k)$ and $S_{\sigma}^{>}(k)$ have the same number of elements.

The lemma follows from the graphical interpretation of the construction of $S_{\sigma}^{>}(k)$ and $S_{\sigma}^{<}(k)$. The graph of $\sigma$ (constructed as in Figure 1) is a sequence of edges connecting the points $(i, \bar{\sigma}(i)$ ). Since the graph starts from $(0,0)$ and since there exists one point with ordinate $n$, any horizontal line with non integer ordinate $y, 0<y<n$, will be crossed from above by a segment (remember the vertical axis is oriented downwards). A similar elementary topological argument shows that such a horizontal line is always crossed successively from above and below by segments, the series of crossings starting from above and ending from below, which implies $\left|S_{\sigma}^{<}(k)\right|=\left|S_{\sigma}^{>}(k)\right|$.

The key step in the proof of convergence is 


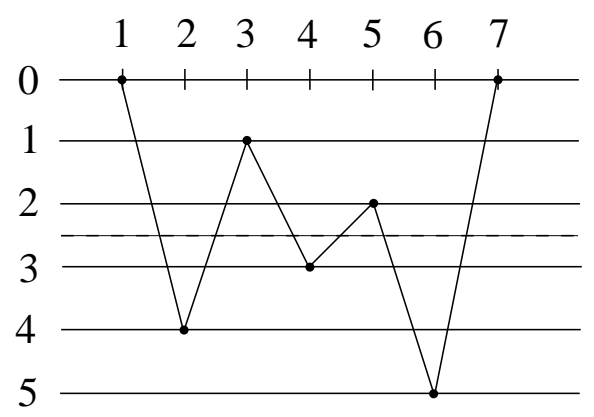

FIG. 2: Construction of $S_{\sigma}^{<}(3)$ for $\sigma=(41325)$. We build $\bar{\sigma}=(0,4,1,3,2,5,0)$, we draw a continuous line $L$ starting from $(1, \bar{\sigma}(1))=(1,0)$ to $(2, \bar{\sigma}(2))=(2,4)$, to $(3, \bar{\sigma}(3))=(3,1), \ldots$, up to $(7, \bar{\sigma}(7))=(7,0)$. To determine $S_{\sigma}^{<}(3)$, we draw a horizontal dashed line just above 3 and we gather the segments of $L$ crossing the dashed line from above. In our case the segments are $((1,0),(2,4)),((3,1),(4,3))$ and $((5,2),(6,5)) . S_{\sigma}^{<}(3)$ is the set of abscissae of the first point of each segment: $S_{\sigma}^{<}(3)=\{1,3,5\}$. Similarly, $S_{\sigma}^{>}(3)$ is obtained from the segments that cross the dashed line from below: $S_{\sigma}^{>}(3)=\{2,4,6\}$.

Lemma 8 For $\sigma \in \mathcal{S}_{n}$ and a given sequence $\alpha_{1}, \ldots, \alpha_{n+1}$, compatible with $\sigma$ (see eq. (10)), we have

$$
X_{\sigma}(k)=k \epsilon+\sum_{j \in S_{\sigma}^{<}(n-k+1)} \imath F_{j}^{Q}-\sum_{j \in S_{\sigma}^{>}(n-k+1)} \imath F_{j}^{P},
$$

where we write $F_{i}^{Q}=F_{i}$ (resp. $F_{i}^{P}=F_{i}$ ) when $\alpha_{i}$ belongs to the image of $Q$ (resp. of $P$ ).

Proof. We first show that it is true for $k=1$. Indeed, $X_{\sigma}(1)=\epsilon+\imath\left(F_{\sigma^{-1}(n)}-F_{\sigma^{-1}(n)+1}\right)$. Let $j=\sigma^{-1}(n)$, we have $\sigma(j)=n$. Then, either $j=1$ and $\left|\alpha_{1}\right\rangle$ is in the image of $Q$, or $j>1$ and $\sigma \in \mathcal{S}_{n}$ implies $\sigma(j)=n>\sigma(j-1)$, so that $\left|\alpha_{j}\right\rangle$ is in the image of $Q$. Thus, in all cases, $F_{\sigma^{-1}(n)}=F_{\sigma^{-1}(n)}^{Q}$. Consider now $F_{j+1}$. Either $j=n$ and $\left|\alpha_{j+1}\right\rangle=\left|\alpha_{n+1}\right\rangle$ is in the image of $P$, or $j<n$ and $\sigma(j+1)<\sigma(j)=n$, so that $\left|\alpha_{j+1}\right\rangle$ is in the image of $P$. Thus, in all cases, $F_{\sigma^{-1}(n)+1}=F_{\sigma^{-1}(n)+1}^{P}$. Therefore, $X_{\sigma}(1)=\epsilon+\imath\left(F_{\sigma^{-1}(n)}^{Q}-F_{\sigma^{-1}(n)+1}^{P}\right)$. On the other hand, $S_{\sigma}^{<}(n)=\left\{\sigma^{-1}(n)\right\}$ and $S_{\sigma}^{>}(n)=\left\{\sigma^{-1}(n)+1\right\}$ since $(j, n)$ is the only point of the graph with ordinate $n$. Thus, the two members of eq. (13) are equal for $k=1$.

Assume now that eq. (13) holds for all the $X_{\sigma}(i)$ with $i=1, \ldots, k, k<n$, and consider the equation (which is true by definition of the $\left.X_{\sigma}(i) \mathrm{s}\right)$ :

$$
X_{\sigma}(k+1)=X_{\sigma}(k)+\epsilon+\imath\left(F_{j}-F_{j+1}\right),
$$

where $j=\sigma^{-1}(n-k)$. We first treat the case $1<j<n$. Four possible situations can arise: (i) $\sigma(j-1)>\sigma(j)>$ $\sigma(j+1)$, (ii) $\sigma(j-1)<\sigma(j)>\sigma(j+1$ ), (iii) $\sigma(j-1)>\sigma(j)<\sigma(j+1)$ and (iv) $\sigma(j-1)<\sigma(j)<\sigma(j+1)$. In case (i), we have $F_{j}=F_{j}^{P}$ and $F_{j+1}=F_{j+1}^{P}$. On the other hand, condition (i) implies $\bar{\sigma}(j)>\bar{\sigma}(j+1)>\bar{\sigma}(j+2)$, so that $S_{\sigma}^{>}(n-k)=S_{\sigma}^{>}(n-k+1)$ and $S_{\sigma}^{<}(n-k)$ is obtained from $S_{\sigma}^{<}(n-k+1)$ by removing $\{j\}$ and adding $\{j+1\}$. eq. (14) together with the hypothesis that eq. (13) holds for $X_{\sigma}(k)$ imply that eq. (13) holds for $X_{\sigma}(k+1)$. Case (ii) implies $F_{j}=F_{j}^{Q}$ and $F_{j+1}=F_{j+1}^{P}$, case (iii) implies $F_{j}=F_{j}^{P}$ and $F_{j+1}=F_{j+1}^{Q}$, case (iv) implies $F_{j}=F_{j}^{Q}$ and $F_{j+1}=F_{j+1}^{Q}$. In all cases, these identities imply that the two expressions (14) and (13) for $X_{\sigma}(k+1)$ do agree.

It remains to treat the boundary cases. If $j=1$, then $F_{j}=F_{1}^{Q}$ and we have either (i) $\sigma(1)<\sigma(2)$ and $F_{2}=F_{2}^{Q}$ or (ii) $\sigma(1)>\sigma(2)$ and $F_{2}=F_{2}^{P}$. We know that $\bar{\sigma}(1)=0$, thus, case (i) corresponds to $\bar{\sigma}(1)<\bar{\sigma}(2)<\bar{\sigma}(3)$, so that according to eq. (13),

$$
X_{\sigma}(k+1)-X_{\sigma}(k)=\imath\left(F_{1}^{Q}-F_{2}^{Q}\right),
$$

in agreement with eq. (14). In case (ii) we have $\bar{\sigma}(1)<\bar{\sigma}(2)>\bar{\sigma}(3)$, which amounts to add $\imath F_{1}^{Q}$ and remove $\imath F_{2}^{P}$. Again, eq. (13) holds for $k+1$. Finally, if $j=n$, then $F_{j+1}=F_{n+1}^{P}$ and we have (i) $\sigma(n-1)<\sigma(n)$ and $F_{n}=F_{n}^{Q}$ or (ii) $\sigma(n-1)>\sigma(n)$ and $F_{n}=F_{n}^{P}$. Case (i) corresponds to $\bar{\sigma}(n)<\bar{\sigma}(n+1)>\bar{\sigma}(n+2)$, case (ii) corresponds to $\bar{\sigma}(n)>\bar{\sigma}(n+1)>\bar{\sigma}(n+2)$. In all cases, the relation given by eq. (13) is satisfied for $k+1$ and the induction proof is complete.

We can now ready to prove 
Theorem 9 The limit

$$
\bar{\Omega}_{\sigma}=\lim _{\epsilon \rightarrow 0} \Omega_{\sigma}(0),
$$

is well-defined.

Proof. To prove the term-wise convergence of $\Omega_{\sigma}(t)$ as $\epsilon \rightarrow 0$, consider eq. (13). The sets $S_{\sigma}^{<}(n-k+1)$ and $S_{\sigma}^{>}(n-k+1)$ have the same number of elements, say $n_{k}$ and, by the gap hypothesis, we have $F_{j}^{Q}-F_{i}^{P} \geq \delta$ for any $i$ and $j$. Therefore, $\left|X_{\sigma}(k)\right|^{2} \geq k^{2} \epsilon^{2}+n_{k}^{2} \delta^{2} \geq \delta^{2}$, since $n_{k} \geq 1$. Thus, the denominator remains away from zero by a finite amount for any $\epsilon \geq 0$ and the limit of $1 / X_{\sigma}(k)$ for $\epsilon \rightarrow 0$ is well-defined.

\section{TREES}

We showed that, for each permutation $\sigma$, the wave operator $\Omega_{\sigma}(t,-\infty)$ has a well-defined limit as $\epsilon \rightarrow 0$. The detailed proof was rather lengthy and the final expression for $X_{\sigma}(k)$ suggests physically the simultaneous occurrence of transitions from states of the model space to states out of it. The convergence is actually much easier to show in terms of trees, and the expressions showing up in the expansion are simpler, mathematically and physically, each factor of the denominator corresponding to a single difference between an energy in the model space and an energy out of it.

In this section, if $N$ is the dimension of the model space, we write $i \in Q$ for $i>N$ and $j \in P$ for $1 \leq j \leq N$, both for notational simplicity, and to emphasize the meaning of the indices, that correspond respectively to eigenstates in the image of $Q$ and in the image of $P$ (i.e. the model space).

Proposition 10 If $T=T_{1} \vee T_{2}$, then, for $t \leq 0$,

$$
\Omega_{T}(t):=\Omega_{T}(t,-\infty)=\sum_{i \in Q, j \in P} \mathrm{e}^{\left(\imath E_{i}^{Q}-\imath E_{j}^{P}+|T| \epsilon\right) t} \Omega_{T}^{i j}\left|\Phi_{i}\right\rangle\left\langle\Phi_{j}\right|,
$$

where $\Omega_{T}^{i j}$ is obtained recursively by:

$$
\begin{aligned}
& \text { For } T=Y, \Omega_{Y}^{i j}:=-\imath \frac{\left\langle\Phi_{i}|V| \Phi_{j}\right\rangle}{\imath E_{i}^{Q}-\imath E_{j}^{P}+\epsilon} . \\
& \text { For } T_{1}=\left|, T_{2} \neq\right|: \Omega_{T}^{i j}:=-\imath \sum_{k \in Q} \frac{\left\langle\Phi_{i}|V| \Phi_{k}\right\rangle \Omega_{T_{2}}^{k j}}{\imath E_{i}^{Q}-\imath E_{j}^{P}+|T| \epsilon} . \\
& \text { For } T_{1} \neq\left|, T_{2}=\right|: \Omega_{T}^{i j}:=\imath \sum_{k \in P} \frac{\Omega_{T_{1}}^{i k}\left\langle\Phi_{k}|V| \Phi_{j}\right\rangle}{\imath E_{i}^{Q}-\imath E_{j}^{P}+|T| \epsilon} . \\
& \text { For } T_{1} \neq\left|, T_{2} \neq\right|: \Omega_{T}^{i j}:=\imath \sum_{k \in P, l \in Q} \frac{\Omega_{T_{1}}^{i k}\left\langle\Phi_{k}|V| \Phi_{l}\right\rangle \Omega_{T_{2}}^{l j}}{\imath E_{i}^{Q}-\imath E_{j}^{P}+|T| \epsilon} .
\end{aligned}
$$

Proof. The computation of $\Omega_{Y}^{i j}$ follows from eq. (12). Let us consider for example the case $T_{1}=\left|, T_{2} \neq\right|$. Then, by applying theorem 5 .

$$
\Omega_{T}(t)=-\imath \sum_{i \in Q, j \in P} \int_{-\infty}^{t} \mathrm{~d} s Q \mathrm{e}^{\epsilon s} \mathrm{e}^{-\imath E_{i}^{Q} s} \mathrm{e}^{\left(\imath E_{i}^{Q}-\imath E_{j}^{P}+\left|T_{2}\right| \epsilon\right) s} \Omega_{T_{2}}^{i j} \mathrm{e}^{i H_{0} s} V\left|\Phi_{i}\right\rangle\left\langle\Phi_{j}\right| .
$$

We replace $Q$ by $\sum_{k \in Q}\left|\Phi_{k}\right\rangle\left\langle\Phi_{k}\right|$ and obtain, by using $|T|=\left|T_{2}\right|+1$,

$$
\begin{aligned}
\Omega_{T}(t) & =-\imath \sum_{k, i \in Q, j \in P} \int_{-\infty}^{t} \mathrm{~d} s \mathrm{e}^{\left(\imath E_{k}^{Q}-\imath E_{j}^{P}+|T| \epsilon\right) s}\left\langle\Phi_{k}|V| \Phi_{i}\right\rangle \Omega_{T_{2}}^{i j}\left|\Phi_{k}\right\rangle\left\langle\Phi_{j}\right| \\
& =-\imath \sum_{k, i \in Q, j \in P} \frac{\mathrm{e}^{\left(\imath E_{k}^{Q}-\imath E_{j}^{P}+|T| \epsilon\right) t}}{\imath E_{k}^{Q}-\imath E_{j}^{P}+|T| \epsilon}\left\langle\Phi_{k}|V| \Phi_{i}\right\rangle \Omega_{T_{2}}^{i j}\left|\Phi_{k}\right\rangle\left\langle\Phi_{j}\right|,
\end{aligned}
$$

The two other cases can be treated similarly.

Since, for arbitrary $i$ and $j, E_{i}^{Q}-E_{j}^{P} \geq \delta$, we get:

Corollary 11 The limit $\bar{\Omega}_{T}(t)=\lim _{\epsilon \rightarrow 0} \Omega_{T}(t)$ is well defined. 


\section{A. Relation with the Rayleigh-Schrödinger perturbation theory}

Kvasnička $^{7}$ and Lindgren ${ }^{6}$ independenty obtained an equation for the time-independent Rayleigh-Schrödinger perturbation theory of possibly degenerate systems:

$$
\left[\bar{\omega}, H_{0}\right] P=V \bar{\omega} P-\bar{\omega} P V \bar{\omega} P,
$$

where the time-independent wave operator $\bar{\omega} P$ transforms eigenstates $\left|\Phi_{0}\right\rangle$ of $H_{0}$ into eigenstates $\bar{\omega} P\left|\Phi_{0}\right\rangle$ of $H_{0}+V$ and where $P \bar{\omega} P=P$ (see ref. 43 p. 202 for details).

Equation (17) is an important generalization of Bloch's classical results $\underline{44}$ because it is also valid for a quasidegenerate model space (i.e. when the eigenstates of $H_{0}$ in the model space have different energies). The relation between time-dependent and time-independent perturbation theory is established by the following proposition:

Proposition 12 We have $\bar{\omega} P=\bar{\Omega}=\lim _{\epsilon \rightarrow 0} \Omega(0)$.

Proof. We take the derivative of eq. (8) with respect to time and we substitute $\chi\left(t, t_{0}\right)=\Omega\left(t, t_{0}\right)-P$. This gives us

$$
\imath \frac{\mathrm{d}}{\mathrm{d} t} \Omega\left(t, t_{0}\right)=H(t) \Omega\left(t, t_{0}\right)-\Omega\left(t, t_{0}\right) H(t) \Omega\left(t, t_{0}\right) .
$$

If we take $t=0$ and $t_{0}=-\infty$, we obtain by continuity

$$
\left.\imath \frac{\mathrm{d}}{\mathrm{d} t} \Omega(t)\right|_{t=0}=V \Omega(0)-\Omega(0) V \Omega(0) .
$$

When we compare this equation with eq. (17), we see that $\bar{\omega} P$ and $\bar{\Omega}$ satisfy the same equation if

$$
\left.\imath \lim _{\epsilon \rightarrow 0} \frac{\mathrm{d} \Omega(t)}{\mathrm{d} t}\right|_{t=0}=\left[\lim _{\epsilon \rightarrow 0} \Omega(0), H_{0}\right] .
$$

To show this, we prove it for each term $\Omega_{T}$. Indeed, eq. (16) gives us

$$
\imath \frac{\mathrm{d} \Omega_{T}(t)}{\mathrm{d} t}=\sum_{i \in Q, j \in P}\left(E_{j}^{P}-E_{i}^{Q}+\imath|T| \epsilon\right) \mathrm{e}^{\left(\imath E_{i}^{Q}-\imath E_{j}^{P}+|T| \epsilon\right) t} \Omega_{T}^{i j}\left|\Phi_{i}\right\rangle\left\langle\Phi_{j}\right|,
$$

and

$$
\left[\Omega_{T}(t), H_{0}\right]=\sum_{i \in Q, j \in P}\left(E_{j}^{P}-E_{i}^{Q}\right) \mathrm{e}^{\left(\imath E_{i}^{Q}-\imath E_{j}^{P}+|T| \epsilon\right) t} \Omega_{T}^{i j}\left|\Phi_{i}\right\rangle\left\langle\Phi_{j}\right| .
$$

By continuity in $\epsilon$, these two expressions are identical for all $t$ when $\epsilon \rightarrow 0$. If we take $t=0$ and we sum over all trees $T$, then we recover eq. (18). Therefore, $\bar{\omega} P$ and $\bar{\Omega}$ satisfy the same equation. It remains to show that they have the same boundary conditions: $\bar{\Omega} P=\bar{\Omega}$ and $P \bar{\Omega}=P$. By eq. (3), these two equations are true for $\Omega(t)$ with any value of $t$ and $\epsilon$.

As a corollary, proposition 10 provides a recursive construction of the wave operator $\bar{\Omega}$.

\section{B. An explicit formula for $\Omega_{T}$}

In this section, we show how $\Omega_{T}(t)$ can be obtained non-recursively from the knowledge of $T$. The key idea is to replace $T$ by another combinatorial object, better suited to that particular computation. We write therefore $\gamma_{T}$ for the smallest permutation for the lexicographical ordering in $S_{T}$ (we view a permutation as a word to make sense of the lexicographical ordering: to (35421) corresponds the word 35421, so that e.g. $(35421)<(54231)$ ). Since $S_{T}$ is always non empty, the map $\gamma: T \longmapsto \gamma_{T}$ is well-defined and an injection from the set of trees to the set of permutations. These permutations are called Catalan permutations $\frac{29}{2}, 312$-avoiding permutations (i.e. permutations for which there does not exist $i<j<k$ such that $\sigma(j)<\sigma(k)<\sigma(i)$, see ref. 31 p. 224), Kempf elements $\stackrel{45}{\underline{y}}$ or stack words $\underline{46}$.

Various elementary manipulations can be done to understand such a map. We list briefly some obvious properties and introduce some notation that will be useful in our forthcoming developments. If $I=\left(a_{1}, \ldots, a_{k}\right)$ is a sequence of integers, we write $I[n]$ for the shifted sequence $\left(a_{1}+n, \ldots, a_{k}+n\right)$. 
Then, let $T=T_{1} \vee T_{2}$ be a tree. The permutation $\gamma(T)$ (that we identify with the corresponding word or sequence) can be constructed recursively as $\gamma(\mid)=\emptyset, \gamma(Y)=(1)$ and

$$
\left.\gamma(T):=\left(\gamma\left(T_{1}\right)[1], 1, \gamma\left(T_{2}\right)\left[\left|T_{1}\right|+1\right]\right)\right) .
$$

The left inverse of $\gamma$, say $\mathcal{T}$, is also easily described recursively as $\mathcal{T}(\emptyset)=\mid, \mathcal{T}(1):=Y$ and

$$
\mathcal{T}(\sigma):=\mathcal{T}((\sigma(1), \ldots, \sigma(k))[-1]) \vee \mathcal{T}((\sigma(k+2), \ldots, \sigma(n))[-k-1]),
$$

where $\sigma \in \mathcal{S}_{n}, \sigma=(\sigma(1), \ldots, \sigma(k), 1, \sigma(k+2), \ldots, \sigma(n))$ is in the image of $\gamma$.

Permutations in the image of $\gamma$ can be characterized recursively similarly: with the same notation as in the previous paragraph, $\sigma$ is in the image of $\gamma$ if and only if $(\sigma(1), \ldots, \sigma(k))[-1]$ and $(\sigma(k+2), \ldots, \sigma(n))[-k-1]$ are in the image of $\gamma$, where $k$ is the integer such that $\sigma(k)=1$,

We are now in a position to compute $\Omega_{T}(t)$. Let us write $\mathcal{A}_{T}$ for all the sequences $\boldsymbol{\alpha}=\left(\alpha_{1}, \ldots, \alpha_{n+1}\right)$ associated to $\gamma(T)$ as in equation (10). We write, as usual, $F_{i}$ for the eigenvalue associated to $\alpha_{i}$. Recall that $\alpha_{1} \in Q, \alpha_{n+1} \in P$ whereas $\alpha_{i}, i \neq 1, n+1$ is in $Q$ if $\sigma(i)>\sigma(i-1)$ and in $P$ otherwise.

These sequences are actually common to the expansions of all the $\Omega_{\sigma}(t), \sigma \in S_{T}$ (this is because they depend only on the positions of descents in the permutations $\sigma \in S_{T}$, as discussed in the proof of thm. (5)). They appear therefore in the expansion of $\Omega_{T}(t)=\sum_{\sigma \in S_{T}} \Omega_{\sigma}(t)$. They actually also correspond exactly to the sequences of eigenvectors that show up in the recursive expansion of $\Omega_{T}(t)$ (proposition 16) (this should be clear from our previous remarks, but can be checked directly from the definition of the recursive expansion). We can refine the recursion of proposition 10 accordingly:

Lemma 13 We have: $\Omega_{T}(t)=\sum_{\boldsymbol{\alpha}} \mathrm{e}^{\left(\imath F_{1}-\imath F_{n+1}+|T| \epsilon\right) t} \Omega_{T}^{\boldsymbol{\alpha}}\left|\alpha_{1}\right\rangle\left\langle\alpha_{n+1}\right|$, where $\Omega_{T}^{\boldsymbol{\alpha}}(t)$ is defined recursively by:

$$
\begin{gathered}
\Omega_{Y}^{\Phi_{i}, \Phi_{j}}=-\imath \frac{\left\langle\Phi_{i}|V| \Phi_{j}\right\rangle}{\imath E_{i}^{Q}-\imath E_{j}^{P}+\epsilon}, \\
\Omega_{T}^{\alpha}=\imath \frac{\Omega_{T_{1}}^{\left(\alpha_{1}, \ldots, \alpha_{k}\right)}\left\langle\alpha_{k}|V| \alpha_{k+1}\right\rangle \Omega_{T_{2}}^{\left(\alpha_{k+1}, \ldots, \alpha_{n}, \alpha_{n+1}\right)}}{\imath F_{1}-\imath F_{n+1}+|T| \epsilon},
\end{gathered}
$$

where $T=T_{1} \vee T_{2}$ and $k=\left|T_{1}\right|$. For $T_{1}=\mid$, we have $\Omega_{T_{1}}^{\left(\alpha_{1}\right)}=-1$ and for $T_{2}=\mid$, we have $\Omega_{T_{2}}^{\left(\alpha_{n+1}\right)}=1$.

Let us now consider $\gamma_{T}$. For $i=1, \ldots, n$, we set: $l(i)=\inf \left\{j \leq i \mid \forall k, j \leq k \leq i, \gamma_{T}(k) \geq \gamma_{T}(i)\right\}$ and $r(i)=\sup \{j \geq$ $\left.i \mid \forall l, j \geq l \geq i, \gamma_{T}(l) \geq \gamma_{T}(i)\right\}$. In words, $l(i)$ is defined as follows: consider all the consecutive positions $k$ on the left of position $i$, such that the value of the permutation $\gamma_{T}(k)$ is larger than $\gamma_{T}(i)$. Then, $l(i)$ is the leftmost of these positions $k$. Similarly, $r(i)$ is the rightmost position $j$ such that, on all positions $k$ between $i$ and $j$, the permutation $\gamma_{T}(k)$ is larger than $\gamma_{T}(i)$.

Theorem 14 We have:

$$
\Omega_{T}^{\boldsymbol{\alpha}}=(-\imath)^{n}(-1)^{d-1}\left\langle\alpha_{1}|V| \alpha_{2}\right\rangle \ldots\left\langle\alpha_{n}|V| \alpha_{n+1}\right\rangle \prod_{i=1}^{n} \frac{1}{\imath F_{l(i)}-\imath F_{r(i)+1}+(r(i)-l(i)+1) \epsilon},
$$

or, equivalently,

$$
\begin{aligned}
\Omega_{T}(t)= & (-\imath)^{n}(-1)^{d-1} \sum_{\alpha}\left|\alpha_{1}\right\rangle\left\langle\alpha_{1}|V| \alpha_{2}\right\rangle \ldots\left\langle\alpha_{n}|V| \alpha_{n+1}\right\rangle\left\langle\alpha_{n+1}\right| \mathrm{e}^{\left(\imath F_{1}-\imath F_{n+1}+|T| \epsilon\right) t} \\
& \prod_{i=1}^{n} \frac{1}{\imath F_{l(i)}-\imath F_{r(i)+1}+(r(i)-l(i)+1) \epsilon},
\end{aligned}
$$

where $d$ is the number of leaves of $T$ pointing to the right.

For example, if $T=Y$, then $\gamma_{T}=(213), l=(113), r=(133)$ and

$$
\Omega_{T}^{\Phi_{i} \Phi_{j} \Phi_{k} \Phi_{l}}=-\imath \sum_{\Phi_{i} \Phi_{j} \Phi_{k} \Phi_{l}} \frac{\left|\Phi_{i}\right\rangle\left\langle\Phi_{i}|V| \Phi_{j}\right\rangle\left\langle\Phi_{j}|V| \Phi_{k}\right\rangle\left\langle\Phi_{k}|V| \Phi_{l}\right\rangle\left\langle\Phi_{l}\right|}{\left(\epsilon+\imath\left(E_{i}^{Q}-E_{j}^{P}\right)\right)\left(\epsilon+\imath\left(E_{k}^{Q}-E_{l}^{P}\right)\right)\left(3 \epsilon+\imath\left(E_{i}^{Q}-E_{l}^{P}\right)\right)} .
$$


Proof. We show that $\Omega_{T}^{\alpha}$ as defined in theorem 14 satisfies the recursion relation of lemma 13, Let us first consider that $T_{1} \neq \mid$ and $T_{2} \neq \mid$. Let $i_{0}$ denote the index such that $\gamma_{T}\left(i_{0}\right)=1$, with $1<i_{0}<n$. The term of the numerator corresponding to $i_{0}$ is $\left\langle\alpha_{i_{0}}|V| \alpha_{i_{0}+1}\right\rangle$, which is the central term of the recursion relation. We have $l\left(i_{0}\right)=1$ and $r\left(i_{0}\right)=n$. Thus, the denominator is $\imath F_{l\left(i_{0}\right)}-\imath F_{r\left(i_{0}\right)+1}+\left(r\left(i_{0}\right)-l\left(i_{0}\right)+1\right) \epsilon=\imath F_{1}-\imath F_{n+1}+n \epsilon$, which is the denominator of the recursion relation. Now we check that the product of terms for $i<i_{0}$ in theorem 14 is $\Omega_{T_{1}}^{\left(\alpha_{1}, \ldots, \alpha_{i_{0}-1}\right)}$. The matrix elements $\left\langle\alpha_{1}|V| \alpha_{2}\right\rangle \ldots\left\langle\alpha_{i_{0}-1}|V| \alpha_{i_{0}}\right\rangle$ obviously agree, so we must check that the denominators agree. Thus, we verify that, for $1 \leq i<i_{0}, l_{T}(i)=l_{T_{1}}(i)$ and $r_{T}(i)=r_{T_{1}}(i)$, where $l_{T}$ and $r_{T}$ denote the $l$ and $r$ vectors for tree $T$. We know that $\gamma_{T}(i)=\gamma_{T_{1}}(i)+1$ for $1 \leq i<i_{0}$. Thus, for $k \leq i, \gamma_{T}(k) \geq \gamma_{T}(i)$ if and only if $\gamma_{T_{1}}(k) \geq \gamma_{T_{1}}(i)$ and $l_{T}(i)=l_{T_{1}}(i)$. For $r_{T}$, we notice that for $l=i_{0}$ we have $\gamma_{T}(l)=1<\gamma_{T}(i)$ and the relation $\gamma_{T}(l) \geq \gamma_{T}(i)$ does not hold. Therefore, $r_{T}(i)<i_{0}$ and $r_{T}(i)=r_{T_{1}}(i)$ by the same argument as $l_{T}(i)=l_{T_{1}}(i)$. The same reasoning holds for $T_{2}$ and the recursion relation is satisfied. The cases $T_{1}=\mid$ or $T_{2}=\mid$ are proved similarly.

We conclude this section with a geometrical translation of the previous theorem. Consider a tree $T$ with $|T|=n$ and number its leaves from 1 for the leftmost leave to $n+1$ for the rightmost one. For each vertex $v$ of $T$, take the subtree $T_{v}$ for which $v$ is the root. In other words, $T_{v}$ is obtained by chopping the edge below $v$ and considering the half-edge dangling from $v$ as the dangling line of the root of $T_{v}$. For each tree $T_{v}$, build the pair $\left(l_{v}, r_{v}\right)$ which are the indices of the leftmost and rightmost leaves of $T_{v}$. Recall that $\left|\alpha_{i}\right\rangle$ belongs to the image of $Q$ (resp. $P$ ) if leaf $i$ points to the left (resp. right): this implies in particular that $F_{l_{v}}=F_{l_{v}}^{Q}$ and $F_{r_{v}}=F_{r_{v}}^{P}$. Then, the set of pairs $\left(l_{v}, r_{v}\right)$ where $v$ runs over the vertices of $T$ is the same as the set of pairs $(l(i), r(i)+1)$ of the theorem. The formula for $\Omega_{T}^{\alpha}$ can be rewritten accordingly and this geometrical version can be proved recursively as theorem 14. Conversely, it can be used to determine the tree corresponding to a given denominator.

\section{CONCLUSION}

We considered three expansions of the wave operator and we proved their adiabatic convergence. We proposed to expand the wave operator over trees, and proved that this expansion reduced the number of terms of the expansion with respect to usual (tractable) ones, simplified the denominators of the expansion into a product of the difference of two energies and lead to powerful formulas and recursive computational methods.

We then showed that this simplification is closely related to the algebraic structure of the linear span of permutations and of a certain convolution subalgebra of trees.

As far as the many-body problem is concerned, we showed that the simplification of diagrams is not due to the details of the Hamiltonian but to the general structure of the wave operator. When the eigenstates and Hamiltonian are expressed in terms of creation and annihilation operators and quantum fields, the algebra of trees mixes with the Hopf algebraic structure of fields 47,48 . It would be interesting to investigate the interplay of these algebraic structures.

The terms of the Rayleigh-Schrödinger series are usually considered to be "quite complicated" (see ref. 49, p. 8) and difficult to work with. The general term of the Rayleigh-Schrödinger series for quasi-degenerate systems is obtained as the limit for $\epsilon \rightarrow 0$ of $\Omega_{T}(0)$ in theorem 14 Through our recursive and non-recursive expressions for these terms, many proofs of their properties become almost trivial. The tree structure suggests various resummations of this series, that will be explored in a forthcoming publication.

\section{Acknowledgments}

This work was partly supported by the ANR HOPFCOMBOP. One of the authors (Â.M.) was supported through the fellowship SFRH/BPD/48223/2008 provided by the Portuguese Science and Technology Foundation (FCT).

\section{Appendix A: A crucial lemma}

In this appendix we state and prove a lemma that is crucial to demonstrate theorem 5 , which is one of the main results of our paper. We first need a noncommutative analogue of Chen's formulas for products of iterated integrals. This analogue, proved in refs. 50 and 22, provides a systematic link between the theory of iterated integrals, the combinatorics of descents, free Lie algebras and noncommutative symmetric functions (see ref. 22 and Appendix B of the present article for further details). 
Let $L=\left(L_{1}, \ldots, L_{n}\right)$ be an arbitrary sequence of time-dependent operators $L_{i}(t)$, satisfying the same regularity conditions as $H(t)$ in section [I] Let $\sigma$ be a permutation in $\mathcal{S}_{n}$ and define:

$$
\Omega_{\sigma}^{L}\left(t, t_{0}\right):=\int_{t_{0}}^{t} \mathrm{~d} t_{1} \int_{t_{0}}^{t_{1}} \mathrm{~d} t_{2} \ldots \int_{t_{0}}^{t_{n-1}} \mathrm{~d} t_{n} L_{1}\left(t_{\sigma(1)}\right) \ldots L_{n}\left(t_{\sigma(n)}\right) .
$$

The notation is extended linearly to combinations of permutations, so that for $\mu:=\sum_{\sigma \in \mathcal{S}_{n}} \mu_{\sigma} \sigma$, with $\mu_{\sigma} \in \mathbb{C}$. Then $\Omega_{\mu}^{L}\left(t, t_{0}\right)$ is defined as the linear combination $\sum_{\sigma \in \mathcal{S}_{n}} \mu_{\sigma} \Omega_{\sigma}^{L}\left(t, t_{0}\right)$. For $K:=\left(K_{1}, \ldots, K_{m}\right)$ another sequence of time-dependent operators, we write $L \cdot K$ for the concatenation product $\left(L_{1}, \ldots, L_{n}, K_{1}, \ldots, K_{m}\right)$.

We also need to define the convolution product of two permutations. If $\alpha \in \mathcal{S}_{n}$ and $\beta \in \mathcal{S}_{m}$, then $\alpha * \beta$ is the sum of the $\left(\begin{array}{c}n+m \\ n\end{array}\right)$ permutations $\gamma \in \mathcal{S}_{n+m}$ such that $\operatorname{st}(\gamma(1), \ldots, \gamma(n))=(\alpha(1), \ldots, \alpha(n))$ and $s t(\gamma(n+1), \ldots, \gamma(n+m))=$ $(\beta(1), \ldots, \beta(m))$. Here, st is the standardization map defined in section IIC1. For instance,

$$
\begin{aligned}
& (2,3,1) *(1)=(2,3,1,4)+(2,4,1,3)+(3,4,1,2)+(3,4,2,1), \\
& (1,2) *(2,1)=(1,2,4,3)+(1,3,4,2)+(1,4,3,2)+(2,3,4,1)+(2,4,3,1)+(3,4,2,1) .
\end{aligned}
$$

In words, the product of two permutations $\alpha \in \mathcal{S}_{n}$ and $\beta \in \mathcal{S}_{m}$ is the sum of all permutations $\sigma$ of $\mathcal{S}_{n+m}$ such that the elements of the sequence $(\sigma(1), \ldots, \sigma(n))$ are ordered as the elements of $(\alpha(1), \ldots, \alpha(n))$, in the sense that $\alpha(i)>\alpha(j)$ if and only if $\sigma(i)>\sigma(j)$ and the elements of $(\sigma(n+1), \ldots, \sigma(n+m))$ are ordered as the elements of $(\beta(1), \ldots, \beta(m))$.

Now, we can state the noncommutative Chen formula 50 (see also remark 3.3, p. 4111 of ref. 22)

Lemma 15 We have:

$$
\Omega_{\alpha}^{L}\left(t, t_{0}\right) \Omega_{\beta}^{K}\left(t, t_{0}\right)=\Omega_{\alpha * \beta}^{L \cdot K}\left(t, t_{0}\right) .
$$

The following lemma can be proven similarly:

Lemma 16 We have, for $L$ and $K$ as above and $J$ a time-dependent operator:

$$
\int_{t_{0}}^{t} \mathrm{~d} s \Omega_{\alpha}^{L}\left(s, t_{0}\right) J(s) \Omega_{\beta}^{K}\left(s, t_{0}\right)=\sum_{\gamma} \Omega_{\gamma}^{L \cdot(J) \cdot K}\left(t, t_{0}\right),
$$

where $\gamma$ runs over the permutations in $\mathcal{S}_{n+m+1}$ with $\gamma(n+1)=1$, st $(\gamma(1), \ldots, \gamma(n))=\alpha$, st $(\gamma(n+2), \ldots, \gamma(n+m+1))=$ $\beta$.

Proof. We expand $\Omega_{\alpha}^{L}$ and $\Omega_{\beta}^{K}$

$$
\begin{aligned}
\int_{t_{0}}^{t} \mathrm{~d} s \Omega_{\alpha}^{L}\left(s, t_{0}\right) J(s) \Omega_{\beta}^{K}\left(s, t_{0}\right)= & \int_{t_{0}}^{t} \mathrm{~d} s \int_{t_{0}}^{s} \mathrm{~d} u_{1} \ldots \int_{t_{0}}^{u_{n-1}} \mathrm{~d} u_{n} \int_{t_{0}}^{s} \mathrm{~d} v_{1} \ldots \int_{t_{0}}^{v_{m-1}} \mathrm{~d} v_{m} \\
& L_{1}\left(u_{\alpha(1)}\right) \ldots L_{n}\left(u_{\alpha(n)}\right) J(s) K_{1}\left(v_{\beta(1)}\right) \ldots K_{m}\left(v_{\beta(m)}\right) .
\end{aligned}
$$

This is the same formula as for the expansion of $\Omega_{\alpha}^{L}\left(s, t_{0}\right) \Omega_{\beta}^{K}\left(s, t_{0}\right)$, up to the term $J(s)$ and the integration $\int_{t_{0}}^{t} \mathrm{~d} s$ that however do not change the underlying combinatorics. Therefore, lemma 15 holds in the form

$$
\begin{aligned}
\int_{t_{0}}^{t} \mathrm{~d} s \Omega_{\alpha}^{L}\left(s, t_{0}\right) J(s) \Omega_{\beta}^{K}\left(s, t_{0}\right)= & \sum_{\sigma} \int_{t_{0}}^{t} \mathrm{~d} s \int_{t_{0}}^{s} \mathrm{~d} s_{1} \ldots \int_{t_{0}}^{s_{n+m-1}} \mathrm{~d} s_{n+m} \\
& L_{1}\left(s_{\sigma(1)}\right) \ldots L_{n}\left(s_{\sigma(n)}\right) J(s) K_{1}\left(s_{\sigma(n+1)}\right) \ldots K_{m}\left(s_{\sigma(n+m)}\right),
\end{aligned}
$$

where, by the definition of $\alpha * \beta$, the sum over $\sigma$ is over all the permutations of $\mathcal{S}_{n+m}$ such that $\operatorname{st}(\sigma(1), \ldots, \sigma(n))=\alpha$ and $s t(\sigma(n+1), \ldots, \sigma(n+m))=\beta$. Now, we change variables to $t_{1}=s, t_{i+1}=s_{i}$ for $i=1, \ldots, n+m$. The permutation $\gamma$ of $t_{1}, \ldots, t_{n+m+1}$ corresponding to the permutation $\sigma$ of $s_{1}, \ldots, s_{n+m}$ is characterized by $\gamma(n+1)=1$ (because $s \geq s_{i}$ for all $i$ ), $\gamma(i)=\sigma(i)+1$ for $1 \leq i \leq n$ and $\gamma(i+1)=\sigma(i)+1$ for $n+1 \leq i \leq n+m$. Therefore,

$$
\begin{aligned}
\int_{t_{0}}^{t} \mathrm{~d} s \Omega_{\alpha}^{L}\left(s, t_{0}\right) J(s) \Omega_{\beta}^{K}\left(s, t_{0}\right)= & \sum_{\gamma} \int_{t_{0}}^{t} \mathrm{~d} t_{1} \ldots \int_{t_{0}}^{t_{n+m}} \mathrm{~d} t_{n+m+1} \\
& L_{1}\left(t_{\gamma(1)}\right) \ldots L_{n}\left(t_{\gamma(n)}\right) J\left(t_{\gamma(n+1)}\right) K_{1}\left(t_{\gamma(n+2)}\right) \ldots K_{m}\left(t_{\gamma(n+m+1)}\right),
\end{aligned}
$$

where $\gamma$ satisfies $\gamma(n+1)=1$, st $(\gamma(1), \ldots, \gamma(n))=\alpha, \operatorname{st}(\gamma(n+2), \ldots, \gamma(n+m+1))=\beta$. The lemma is proved.

Notice that the same process would allow to derive combinatorial formulas for arbitrary products of iterated integrals and for integrals with integrands involving iterated integrals, provided these products and integrands have expressions similar to the ones considered in the two lemmas. 


\section{Appendix B: The algebraic structure of tree-shaped iterated integrals}

Our exposition of tree-parametrized time-dependent perturbation theory has focussed on the derivation of convergence results and explicit formulas for the time-dependent wave operator. However, the reasons why such an approach is possible and efficient are grounded into various algebraic and combinatorial properties of trees, descents and similar objects.

These properties suggest that the theory of effective Hamiltonians is grounded into a new "Lie theory" generalizing the usual Lie theory (or, more precisely, generalizing the part of the classical Lie theory that is relevant to the study of the solutions of differential equations). First indications that such a theory exists were already pointed out in our ref. 22. Indeed, we showed in this article that descent algebras of hyperoctahedral groups and generalizations thereof are relevant to the time-dependent perturbation theory. Applications included an extension of the Magnus expansion for the time-dependent wave operator. Our results below provide complementary insights on the subject and further evidence that algebraic structures underly many-body theories.

\section{The algebra structure}

We know from ref. 22 and Sect IIB that the family of integrals $\Omega_{\sigma}^{L}\left(t, t_{0}\right)$ is closed under the product. This closure property is reflected into the convolution product of permutations. This result is a natural noncommutative generalization of Chen's formula for the product of iterated integrals. We show, in the present section, that the same result holds for integrals parametrized by trees. We will explain, in the next sections, why such a result -which may seem surprising from the analytical point of view- could be expected from the modern theory of combinatorial Hopf algebras.

For $L=\left(L_{1}, \ldots, L_{n}\right)$ a family of time-dependent operators (with the usual regularity conditions), and $T$ a tree with $n$ internal vertices, we write

$$
\Omega_{T}^{L}\left(t, t_{0}\right):=\sum_{\sigma \in S_{T}} \int_{t_{0}}^{t} \mathrm{~d} t_{1} \int_{t_{0}}^{t_{1}} \mathrm{~d} t_{2} \ldots \int_{t_{0}}^{t_{n-1}} \mathrm{~d} t_{n} L_{1}\left(t_{\sigma(1)}\right) L_{2}\left(t_{\sigma(2)}\right) \ldots L_{n}\left(t_{\sigma(n)}\right) .
$$

This notation is extended to linear combinations of trees, so that e.g. if $Z=T+2 T^{\prime}$, where $T$ and $T^{\prime}$ are two arbitrary trees with the same number of vertices, then $\Omega_{Z}^{L}\left(t, t_{0}\right)=\Omega_{T}^{L}\left(t, t_{0}\right)+2 \Omega_{T^{\prime}}^{L}\left(t, t_{0}\right)$. For $i \leq n$, we write $L_{\leq i}=\left(L_{1}, \ldots, L_{i}\right)$, $L_{\geq i}=\left(L_{i}, \ldots, L_{n}\right)$.

Proposition 17 For $L=\left(L_{1}, \ldots, L_{n}\right)$ and $K=\left(K_{1}, \ldots, K_{m}\right)$ two families of time-dependent operators and $T=T_{1} \vee T_{2}$, $U=U_{1} \vee U_{2}$ two trees, $|T|=n,\left|T_{1}\right|=p,\left|T_{2}\right|=q,|U|=m,\left|U_{1}\right|=l,\left|U_{2}\right|=k$, we have:

$\Omega_{T}^{L}\left(t, t_{0}\right) \Omega_{U}^{K}\left(t, t_{0}\right)=\int_{t_{0}}^{t} d s \Omega_{T}^{L}\left(s, t_{0}\right) \Omega_{U_{1}}^{K_{\leq l}}\left(s, t_{0}\right) K_{l+1}(s) \Omega_{U_{2}}^{K_{\geq l+2}}\left(s, t_{0}\right)+\int_{t_{0}}^{t} d s \Omega_{T_{1}}^{L_{\leq p}}\left(s, t_{0}\right) L_{p+1}(s) \Omega_{T_{2}}^{L_{\geq p+2}}\left(s, t_{0}\right) \Omega_{U}^{K}\left(s, t_{0}\right)$.

In the formula, one or several of the trees $T_{1}, T_{2}, U_{1}, U_{2}$ may be the trivial tree $\mid$.

Proof. Recall the integration by parts formula. For any integrable functions $f$ and $g$ we define $F(t):=\int_{t_{0}}^{t} \mathrm{~d} s f(s)$, $G(t):=\int_{t_{0}}^{t} \mathrm{~d} s g(s)$ and $H(t):=F(t) G(t)$. Then

$$
H(t)=\int_{t_{0}}^{t} \mathrm{~d} s \frac{\mathrm{d} H(s)}{\mathrm{d} s}=\int_{t_{0}}^{t} \mathrm{~d} s f(s) G(s)+\int_{t_{0}}^{t} \mathrm{~d} s F(s) g(s) .
$$

Now, we use this identity with $F(t)=\Omega_{T}^{L}\left(t, t_{0}\right)$. It follows from the proof of theorem 5 that $f(s)$ is given by

$$
f(s)=\Omega_{T_{1}}^{L_{\leq p}}\left(s, t_{0}\right) L_{p+1}(s) \Omega_{T_{2}}^{L_{\geq p+2}}\left(s, t_{0}\right),
$$

with a similar identity for $G(t)=\Omega_{U}^{K}\left(t, t_{0}\right)$. The proposition follows.

In particular, it is a consequence of the proposition and a straightforward recursion argument that the linear span of the integrals $\Omega_{T}^{L}\left(t, t_{0}\right)$ is closed under products. 


\section{Hopf algebras and Lie theory}

This result may be formalized algebraically. Let us write $\mathcal{T}$ for the set of formal power series with complex coefficients over the set of trees. Proposition 17 enables us to define a product on trees, denoted by $*$, such that $\Omega_{T}^{L}\left(t, t_{0}\right) \Omega_{U}^{K}\left(t, t_{0}\right)=\Omega_{T * U}^{L \cdot K}\left(t, t_{0}\right)$. This product is defined recursively by the equation ${ }^{30}$

$$
T * U:=\left(T * U_{1}\right) \vee U_{2}+T_{1} \vee\left(T_{2} * U\right) .
$$

Proof. The empty tree $\mid$ is the unit for the product $*$. Assume that $T * U$ is defined and satisfies the recursive relation for all trees such that $|T|+|U|<n$, and consider two trees $T$ and $U$ with $|T|+|U|=n$. The first term on the right hand side of proposition 17 is $\int_{t_{0}}^{t} d s \Omega_{T}^{L}\left(s, t_{0}\right) \Omega_{U_{1}}^{K_{\leq l}}\left(s, t_{0}\right) K_{l+1}(s) \Omega_{U_{2}}^{K_{\geq l+2}}\left(s, t_{0}\right)$. By the recursive relation, we have

$$
\Omega_{T}^{L}\left(s, t_{0}\right) \Omega_{U_{1}}^{K \leq l}\left(s, t_{0}\right)=\Omega_{T * U_{1}}^{L \cdot K_{\leq l}} .
$$

Thus, the whole term can be written

$$
\int_{t_{0}}^{t} d s \Omega_{T}^{L}\left(s, t_{0}\right) \Omega_{U_{1}}^{K_{\leq l}}\left(s, t_{0}\right) K_{l+1}(s) \Omega_{U_{2}}^{K_{2 l+2}}\left(s, t_{0}\right)=\Omega_{\left(T * U_{1}\right) \vee U_{2}}^{L \cdot K} .
$$

The second term of proposition 17 is treated similarly and we obtain

$$
\Omega_{T}^{L}\left(t, t_{0}\right) \Omega_{U}^{K}\left(t, t_{0}\right)=\Omega_{\left(T * U_{1}\right) \vee U_{2}}^{L \cdot K}+\Omega_{\left(T_{1} \vee\left(T_{2} * U\right)\right.}^{L \cdot K}
$$

Therefore, the relation $\Omega_{T}^{L}\left(t, t_{0}\right) \Omega_{U}^{K}\left(t, t_{0}\right)=\Omega_{T * U}^{L \cdot K}\left(t, t_{0}\right)$ gives us

$$
T * U=\left(T * U_{1}\right) \vee U_{2}+T_{1} \vee\left(T_{2} * U\right) .
$$

Corollary 18 The product provides $\mathcal{T}$ with the structure of an associative algebra.

The corollary is a by-product of the associativity of the product of operators and of proposition 17

Of course, although the analysis of tree-shaped iterated integrals leads to a straightforward proof, the associativity property is a purely combinatorial phenomenon that originates ultimately from the associativity of the shuffle product. See ref. 22 for the connections between the noncommutative Chen formula and shuffle products, see also section 4 of ref. 51 for Schützenberger's classical (but rarely quoted) analysis of the formal properties of the shuffle product -in fact, the splitting of the convolution product of trees reflects the classical splitting of shuffle products into left and right half-shuffle products that had appeared in the study of Lie polynomials and was first encoded combinatorially in ref. 51. The associativity can also be checked directly or deduced from the associativity of the convolution product $*$ of permutations, since one may verify easily, using e.g. our description of the permutations in $S_{T}$ that the convolution product as introduced above is nothing but the restriction to $\mathcal{T}$ of the convolution product on the algebra $\mathcal{S}=$ $\prod_{n \in \mathbf{N}} \mathbf{C}\left[\mathcal{S}_{n}\right]$.

This fact that the linear span of trees (often written PBT) defines a subalgebra of $\mathcal{S}$ for the convolution product (and even a Hopf subalgebra, referred to as the Hopf algebra of planar binary trees, whereas $\mathcal{S}$ is referred to as the Malvenuto-Reutenauer or Hopf algebra of free quasi-symmetric functions in the litterature) is well-known. It was first observed in ref. 30 and further investigated in a series of papers $34,36,37.52$. There is however a slight subtelty here, since the embedding of PBT in $\mathcal{S}$ considered e.g. in ref. 30 is not the one we consider (another projection map from permutations to trees is used) so that PBT and $\mathcal{T}$, although isomorphic as algebras (the product rule in PBT is the same as the one in $\mathcal{T}$, see e.g. proposition 3.2 of ref. 30 , where $\mathbf{P B T}$ is written $\mathbf{C}\left[Y_{\infty}\right]$ ) do not agree as subalgebras of $\mathcal{S}$.

However, a corollary of this isomorphism is that the structures existing on PBT carry over to the analysis of the algebraic properties of tree-shaped iterated integrals. The existence of a Hopf algebra structure seems particularly meaningful since the classical applications of the theory of free Lie algebras to the analysis of differential equations can be rewritten using the framework of Hopf algebras (see e.g. the accounts in refs. 53, 54).

The same observation holds for the direct sum of the hyperoctahedral group algebras, that also carries naturally a Hopf algebra structure 5 , 56 , and which applications to time-dependent perturbative Hamiltonians were studied in ref. 22. We leave for further research the investigation of the possible role of these Hopf algebra structures in the context of many-body theories. 


\section{Permutations, trees and descents}

Another meaningful observation, along the same lines, is that the three expansions we derived, based respectively on sequences of $P$ and $Q$ (as in the first expression for $\Omega\left(t, t_{0}\right)$ ), on trees (third expression) and on permutations (second expression), reflect at the analytical level the existence of projection maps and embeddings between hypercubes (or the descent algebra), planar binary trees, and permutations. Following Viennot ${ }^{23}$, these maps have been at the origin of modern enumerative combinatorics. We refer to refs. $30,34,36,37$ for a detailed study of these maps that emphasizes the existence of underlying geometrical structures that go beyond the Hopf algebraic ones.

1 T. T. S. Kuo and E. Osnes. Folded-Diagram Theory of the Effective Interaction in Nuclei, Atoms and Molecules, volume 364 of Lecture Notes in Physics. Springer Verlag, Berlin, 1990.

2 T. Morita. Perturbation theory for degenerate problems of many-fermion systems. Prog. Theor. Phys., 29:351-69, 1963.

${ }^{3}$ G. Jolicard. A recursive solution to the time dependent Schrödinger equation using a generalized quasidegenerate Bloch formalism. J. Chem. Phys., 90:2320-7, 1989.

${ }^{4}$ G. Jolicard and J. P. Killingbeck. The Bloch wave operator: generalizations and applications: II. The time-dependent case. J. Phys. A: Math. Gen., 36:R411-R473, 2003.

5 W. Pauli. Selected Topics in Field Quantization. Dover, New York, 1973.

${ }^{6}$ I. Lindgren. The Rayleigh-Schrödinger perturbation and the linked-diagram theorem for a multi-configurational model space. J. Phys. B: At. Mol. Phys., 7:2441-70, 1974.

7 V. Kvasnička. Construction of model Hamiltonians in framework of Rayleigh-Schrödinger perturbation theory. Czechoslovak J. Phys., 24:605-15, 1974.

8 R. Gurau, J. Magnen, and V. Rivasseau. Tree quantum field theory. Ann. Henri Poincaré, 10:867-91, 2009.

${ }^{9}$ H. Maassen and D. Botvich. A Galton-Watson estimate for Dyson series. Ann. Henri Poincaré, 10:1141-58, 2009.

10 When $H_{0}$ is quadratic, the time-dependence of the annihilation operators is simply $a_{n} \mathrm{e}^{-\imath \epsilon_{n} t}$, where $\epsilon_{n}$ is the energy of the $n$th eigenvalue of $H_{0}$ (see, e.g. ref. 42). When $H_{0}$ is not quadratic, the time-dependent annihilation operators and the free Feynman propagator cannot be calculated explicitly.

11 L. N. Bulaevskii. Quasihomopolar electron levels in crystals and molecules. J. Exp. Theor. Phys., 24:154-60, 1967.

12 R. DelSole and E. Fiorino. Macroscopic dielectric tensor at crystal surfaces. Phys. Rev. B, 29:4631-45, 1984.

13 D. Foata and M.-P. Schützenberger. Nombres d'Euler et permutations alternantes, 1971. Unpublished manuscript available at www.emis.de/journals/SLC/books/foaschuetz1.pdf.

${ }^{14}$ G. Viennot. Interprétations combinatoires des nombres d'Euler et de Genocchi. Semin. Theor. Nombres (Univ. Bordeaux), 11:1-94, 1981.

15 P. Leroux, F. Bergeron, and G. Labelle. Combinatorial Species and Tree-like Structures, volume 67 of Encyclopedia of Mathematics and its Applications. Cambridge University Press, Cambridge, 1998.

16 This assumption can be relaxed by using the interaction picture (see ref. 17, p. 283 and 38 , p. 557). In the adiabatic limit, we recover the Rayleigh-Schrödinger series, which is valid when the perturbation $V$ is relatively-bounded with respect to $H_{0}$ (see Chapter XII of ref. 49).

17 M. Reed and B. Simon. Methods of Modern Mathematical Physics. II Fourier Analysis, Self-adjointness. Academic Press, New York, 1975.

18 C. Bloch. Sur le détermination de l'état fondamental d'un système de particules. Nucl. Phys., 7:451-8, 1958.

19 M. A. J. Michels and L. G. Suttorp. Diagrammatic analysis of adiabatic and time-independent perturbation theory for degenerate energy levels. Physica A, 58:385-8, 1978.

${ }^{20} \mathrm{Yu}$. Dmitriev and O. V. Solnyshkina. Adiabatic perturbation theory for the degenerated case. III. Adiabatic formalism by Gell-Mann and Low with an arbitrary switching function. Int. J. Quant. Chem., 33:543-553, 1988.

21 I. Lindgren, S. Salomonson, and B. Åsén. The covariant-evolution-operator method for bound-state QED. Phys. Repts., 389:161-261, 2004.

${ }^{22}$ Ch. Brouder and F. Patras. Hyperoctahedral Chen calculus for effective Hamiltonians. J. Algebra, 322:4105-20, 2009.

23 G. Viennot. Permutations ayant une forme donnée. Discrete Math., 26:279-84, 1979.

24 L. M. Frantz and R. L. Mills. Many-body basis for the optical model. Nuclear Phys., 15:16-32, 1960.

${ }^{25}$ H. A. Bethe, B. H. Brandow, and A. G. Petscheck. Reference spectrum method for nuclear matter. Phys. Rev., 129:225-64, 1963.

${ }^{26}$ B. H. Brandow. Linked-cluster expansions for the nuclear many-body problem. Rev. Mod. Phys., 39:771-828, 1967.

27 S. Olszewski. Combinatorial analysis of the Rayleigh-Schrödinger perturbation theory based on a circular scale of time. Inter. J. Quant. Chem., 97:784-801, 2004.

28 R. Simion. Noncrossing partitions. Discrete Math., 217:367-409, 2000.

29 A. Panayotopoulos and S. Sapounakis. On binary trees and permutations. Math. Sci. Hum., 117:61-70, 1992.

30 J.-L. Loday and M. O. Ronco. Hopf algebra of the planar binary trees. Adv. Math., 139:293-309, 1998.

31 R. P. Stanley. Enumerative Combinatorics. Volume II. Cambridge University Press, Cambridge, 1986.

32 D. Rawlings. The ABC's of classical enumeration. Ann. Sci. Math. Quebec, 10:207-35, 1986. 
33 R. P. Stanley. Enumerative Combinatorics. Volume I. Cambridge University Press, Cambridge, 1986.

34 J.-L. Loday and M.O. Ronco. Order structure on the algebra of permutations and planar binary trees. J. Algebraic Combin., $15: 253-70,2002$.

35 R.P. Stanley. Theory and applications of plane partitions: Part 1. Stud. Appl. Math., 50:167-88, 1971.

36 F. Chapoton. Algèbres de Hopf des permutoèdres, associaèdres et hypercubes. Adv. Math., 150:264-75, 2000.

37 F. Chapoton. Bigèbres différentielles graduées associées aux permutoèdres, associaèdres et hypercubes. Ann. Inst. Fourier, 50:1127-53, 2000.

38 T. Kato. Perturbation Theory for Linear Operators. Springer Verlag, Berlin, second edition, 1995.

39 Ch. Brouder, G. Panati, and G. Stoltz. Gell-Mann and Low formula for degenerate unperturbed states. Ann. Henri Poincaré, 11:1285-309, 2010.

40 Ch. Brouder, G. Panati, and G. Stoltz. Many-body Green function of degenerate systems. Phys. Rev. Lett., 103:230401, 2009.

41 J. Goldstone. Derivation of the Brueckner many-body theory. Proc. Roy. Soc. London, A239:267-79, 1957.

42 E. K. U. Gross, E. Runge, and O. Heinonen. Many-Particle Theory. Adam Hilger, Bristol, 1991.

43 I. Lindgren and J. Morrison. Atomic Many-Body Theory. Springer, Berlin, second edition, 1986.

44 C. Bloch. Sur la théorie des perturbations des états liés. Nucl. Phys., 6:329-47, 1958.

45 A. Postnikov and R. P. Stanley. Chains in the Bruhat order. J. Algebraic Combin., 29:133-74, 2009.

46 T. Feil, K. Hutson, and R. M. Kretchmar. Tree traversal and permutations. Congressus Numerantium, 172:201-21, 2005.

47 Ch. Brouder, B. Fauser, A. Frabetti, and R. Oeckl. Quantum field theory and Hopf algebra cohomology. J. Phys. A: Math. Gen., 37:5895-927, 2004.

${ }^{48}$ Ch. Brouder. Quantum field theory meets Hopf algebra. Math. Nachr., 282:1664-90, 2009.

49 M. Reed and B. Simon. Methods of Modern Mathematical Physics. IV Analysis of Operators. Academic Press, New York, 1978.

50 A. A. Agrachev and R. V. Gamkrelidze. The shuffle product and symmetric groups. In K. D. Elworthy, W. N. Everitt, and E. B. Lee, editors, Differential Equations, Dynamical Systems and Control Science, volume 152 of Lecture Notes in Pure and Applied Mathematics, pages 365-82. Marcel Dekker, Inc., New York, 1994.

${ }^{51}$ M. P. Schützenberger. Sur une propriété combinatoire des algèbres de lie libres pouvant être utilisée dans un problème de mathématiques appliquées. Séminaire Dubreil-Jacotin Pisot (Algèbre et théorie des nombres), Paris, $1958 / 59$.

52 J.-Y. Thibon, F. Hivert, and J.-C. Novelli. The algebra of binary search trees. Theor. Comput. Sci., 339:129-65, 2005.

53 C. Reutenauer. Free Lie Algebras. Oxford University Press, Oxford, 1997.

${ }^{54}$ F. Patras. L'algèbre des descentes d'une bigèbre graduée. J. Alg., 170:547-66, 1994.

55 M. Aguiar, N. Bergeron, and K. Nyman. The peak algebra and the descent algebras of type B and D. Trans. Amer. Math. Soc., 356:2781-824, 2004 .

56 M. Aguiar, J.-C. Novelli, and J.-Y. Thibon. Unital versions of the higher order peak algebras, 2008. arXiv:0810.4634v1. 